\title{
Development and assessment of a practical stiffness reduction method for the in-plane design of steel frames
}

\author{
Merih Kucukler ${ }^{\mathrm{a}, \mathrm{b}, *}$, Leroy Gardner ${ }^{\mathrm{a}}$, Lorenzo Macorini ${ }^{\mathrm{a}}$ \\ ${ }^{a}$ Department of Civil and Environmental Engineering, South Kensington Campus, Imperial College \\ London, London, SW7 2AZ, UK \\ ${ }^{b}$ Department of Civil Engineering, Bilecik Seyh Edebali University, Bilecik, 11210, Turkey
}

\begin{abstract}
In this paper, the development and assessment of a stiffness reduction method for the inplane design of steel frames is presented. The adopted stiffness reduction approach is implemented by reducing the flexural stiffnesses $(E I)$ of the members of a steel frame by considering the first-order forces they are subjected to through the stiffness reduction functions and performing Geometrically Nonlinear Analysis (i.e. second-order elastic analysis). Since the presented approach uses stiffness reduction functions that fully take into account the deleterious influence of imperfections and the spread of plasticity on the structural response and member strengths, it obviates the need of using member design equations, and only requires cross-section strength checks. The accuracy of the presented approach is illustrated for individual steel members and non-redundant and redundant benchmark steel frames from the literature. In all the considered cases, the presented method is verified against the results obtained from nonlinear finite element modelling. A comparison of the presented approach against the notional load method of the European structural steel design code EN 1993-1-1 and the direct analysis method of the US structural steel design code AISC $360-10$ is also provided.
\end{abstract}

Keywords: Stiffness reduction; steel frames; steel beam-columns; inelastic buckling; imperfections; residual stresses

\section{Introduction}

The development and spread of plasticity, aggravated by the presence of residual stresses and geometrical imperfections, causes the stiffness of steel structures to be significantly eroded under applied loading, which detrimentally influences their stability and strength. In conventional structural steel design carried out in accordance with the current steel design specifications [1-3], the influence of these adverse effects is typically taken into account

\footnotetext{
* Corresponding author

Email addresses: merihkucukler@gmail.com (Merih Kucukler), leroy.gardner@imperial.ac.uk (Leroy Gardner), l.macorini@imperial.ac.uk (Lorenzo Macorini)
} 
through semi-empirical member design equations and adopting the effective length or notional load concepts. Though it is rather straightforward to apply, this type of structural design approach is quite an indirect way of considering the effects of plasticity and imperfections on the response of steel structures. It also generally ignores the changes of forces and moments within the structural elements due to the different extents of stiffness reduction (i.e. plasticity) experienced.

To consider the detrimental influence of the spread of plasticity on the response of a steel structure in a more direct manner, the reduction of stiffness of its members on the basis of the forces and moments they withstand has been recommended by Liew et al. [4, 5], Ziemian and McGuire [6] and Zubydan [7, 8]. These proposals do however require the explicit modelling of the initial member out-of-straightnesses, which can be a quite tedious process due to the need to determine the most unfavourable shape and direction of imperfection for each single member under various loading conditions. Kim and Chen $[9,10]$ proposed the application of further stiffness reduction to the stiffness reduction scheme of $[4,5]$, thereby taking into account the influence of the member out-of-straightness on the behaviour and eliminating the need for its modelling. Nevertheless, this method is based on the refined plastic hinge analysis approach requiring specific software which is not widely available in practice. Surovek-Maleck and White $[11,12]$ put forward a method that can be applied through conventional structural analysis software by performing Geometrically Nonlinear Analysis with reduced stiffness, thereby considering the influence of the spread of plasticity on the structural response and eliminating the need to use sway buckling mode effective lengths in the determination of the strengths of the steel members. With minor changes, this method has now been included in the two most recent versions of the AISC 360 specification, including AISC 360-10 [2], where it is referred to as the direct analysis method. However, since the stiffness reduction scheme of the direct analysis method does not fully consider the erosion of strength in imperfect inelastic columns, it still requires the use of column strength equations in design. Moreover, it leads to overly-conservative results in some cases as it disregards the influence of bending moment shape on the development of plasticity.

In Kucukler et al. [13], a stiffness reduction method for the in-plane design of structural steel members is proposed. Stiffness reduction functions capable of taking into account fully the deleterious influence of the spread of plasticity, residual stresses and geometrical imperfections and of considering the influence of bending moment shape on the development of plasticity were developed. Unlike the direct analysis method of AISC 360-10 [2], the stiffness reduction method of [13], which was verified against the results obtained from nonlinear finite element modelling for a wide range of cases, eliminated the need of using column strength equations, requiring only cross-section strength checks. However, Kucukler et al. [13] only focused on the assessment of the accuracy of their method for individual members and did not apply it to the design of steel frames.

The current paper extends the stiffness reduction method of Kucukler et al. [13], which applies to individual elements, to the design of steel frames. The assessment and verification of the stiffness reduction approach against nonlinear finite element modelling is presented for a series of steel frames. In the proposed approach, Geometrically Nonlinear Analysis 
of the structure, whose members' flexural stiffnesses $(E I)$ have been reduced on the basis of the forces they are resisting in a prior first-order analysis, is performed. The internal forces determined through the Geometrically Nonlinear Analysis are then used to carry out cross-section checks. Provided the ultimate cross-section strength is not exceeded within the structure, the design is satisfactory. Since the influence of the spread of plasticity and imperfections are fully accounted for by stiffness reduction, there is no need to use column strength equations. The proposed approach also does not require the modelling of member out-of-straightnesses, thus removing the need to identify their most detrimental shape and direction. The stiffness reduction approach presented herein is intended to be used with that proposed for out-of-plane design in Kucukler et al. [14, 15] within a design framework based on the separation of the in-plane and out-of-plane analyses of steel frames subjected to in-plane loading.

In the following sections of this paper, the application and accuracy of the presented stiffness reduction method are illustrated by comparing its results against those obtained from Geometrically and Materially Nonlinear Analysis with Imperfections (GMNIA) of finite element models for individual steel members and non-redundant and redundant benchmark steel frames from the literature. To assess the qualities and limitations of the proposed method against other structural steel design approaches, comparisons are also made with the notional load method of EN 1993-1-1 [1] and the direct analysis method of AISC 360-10 [2], both of which require individual member buckling checks.

\section{Finite element modelling}

In this section, the details of the finite element modelling approach adopted in this study are presented. In the subsequent sections, the finite element models, which account for geometrical and material nonlinearities and include geometrical imperfections and residual stresses, are used to verify the proposed stiffness reduction method.

\subsection{Development of finite element models}

In this study, beam elements were employed to create finite element models through the finite element analysis software Abaqus [16]. The linear Timoshenko beam element referred to as B31OS in the Abaqus element library and capable of considering shear deformations and the warping degree of freedom is used in all the numerical simulations. Thirty-three integration points were used within each flange and web of an I section so that the stress and strain variations within the cross-section and the spread of plasticity could be accurately captured. The default Simpson integration method with one integration point located in the middle of an element was chosen for the numerical integration along the element length. The tri-linear elastic-plastic stress-strain relationship shown in Fig. 1 was employed, where $E$ is the Young's modulus, $E_{s h}$ is the strain hardening modulus, $f_{y}$ and $\epsilon_{y}$ are the yield stress and strain respectively and $\epsilon_{s h}$ is the strain value at the onset of strain hardening. The parameters $f_{u}$ and $\epsilon_{u}$ correspond to the ultimate stress and strain respectively. $E_{s h}$ was assumed to be $2 \%$ of $E$ and $\epsilon_{s h}$ was taken as $10 \epsilon_{y}$, conforming to the ECCS recommendations [17] for hot-rolled structural steel. The von Mises yield criterion with associated plastic 
flow and isotropic hardening were assumed in the finite element models. Unless otherwise indicated, grade S235 steel was used in all the simulations.

The ECCS [17] residual stress patterns illustrated in Fig. 2 were applied to the finite element models by defining the initial stress values at the section integration points through the SIGINI user subroutine [16]. The initial member out-of-straightness was assumed to be a half-sine wave in shape and 1/1000 of the corresponding member length in magnitude, while the initial frame out-of-plumbness was assumed to be $0.002 \mathrm{rad}$ [18]. These geometrical imperfections were defined in the most unfavourable directions considering the loading conditions in all the numerical simulations: the initial out-of-straightness was modelled in the direction that the member deforms for single curvature bending cases and mostly deforms for double curvature bending cases, while the initial out-of-plumbness of the frame was modelled in the direction of sway deformation. It is worth noting that for the investigated highly redundant sway frames, it was observed that the out-of-plumbness was the dominant geometrical imperfection in comparison to the member out-of-straightness, which is in accordance with the observations of Ziemian et al. [19] and Maleck [20]. In the finite element models, 100 elements were used to model each individual member of the considered frames. Since this paper investigates the application of the proposed stiffness reduction approach to the in-plane design of steel frames, all the finite element models were fully restrained in the out-of-plane direction.

\subsection{Validation of finite element models}

In Kucukler [21], a series of steel frame and individual member experiments from the literature were employed to verify the finite element modelling approach adopted in this paper. A wide range of validation studies was performed where it was observed that the finite element models are able to replicate accurately the actual response of steel frames and members influenced by geometrical and material nonlinearities and imperfections.

\section{Description of the stiffness reduction method}

This section describes the application of the proposed stiffness reduction method to steel frames. Initially, stiffness reduction functions developed by Kucukler et al. [13] are briefly presented. Then the adopted ultimate cross-section strength equation is provided. Finally, the steps followed in the application of the proposed stiffness reduction method to steel frames are described.

\subsection{Stiffness reduction functions}

This subsection presents the stiffness reduction functions adopted in this study. In the following sections of this paper, these functions will be utilised in the application of the proposed stiffness reduction method to the design of steel frames. 


\subsubsection{Stiffness reduction function for axial loading $\tau_{N}$}

In Kucukler et al. [13], a stiffness reduction function for axial loading $\tau_{N}$ was derived using the European column buckling curves [1]. This stiffness reduction function is given by:

$$
\begin{array}{r}
\tau_{N}=\frac{4 \psi^{2}}{\alpha^{2} N_{E d} / N_{p l}\left[1+\sqrt{1-4 \psi \frac{N_{E d} / N_{p l}-1}{\alpha^{2} N_{E d} / N_{p l}}}\right]^{2}} \text { but } \tau_{N} \leq 1 \\
\text { where } \psi=1+0.2 \alpha \frac{N_{E d}}{N_{p l}}-\frac{N_{E d}}{N_{p l}},
\end{array}
$$

in which $N_{E d}$ is the axial load applied to the member, $N_{p l}$ is the axial yield load equal to the cross-sectional area $A$ multiplied by the yield strength $f_{y}$ (i.e. $N_{p l}=A f_{y}$ ) and $\alpha$ is the imperfection factor. The values of the imperfection factors $\alpha$ for hot-rolled I-section members are provided in Table 1 for the case where failure of the member is about the major axis. Note that in the table, $h$ and $b$ are the overall depth and width of the cross-section of the member. The stiffness reduction function $\tau_{N}$ is used to reduce the flexural stiffnesses of columns; a linear buckling analysis on a column with this reduced stiffness provides an exact match to the EN 1993-1-1 [1] column buckling curves. However, Kucukler [21] observed that the use of $\alpha=0.26$ instead of the imperfection factor value $\alpha=0.34$ recommended by EN 1993-1-1 [1] leads to more accurate results for columns with cross-sections whose aspect ratios are smaller than 1.2 (i.e. $h / b \leq 1.2$ ) and failing about the major axis. Thus, the use of $\alpha=0.26$ for steel members with wide-flange cross-sections (i.e. $h / b \leq 1.2$ ) and undergoing major axis failure is recommended herein, though $\alpha=0.34$ can be used within $\tau_{N}$, resulting in the slightly conservative column strength predictions obtained through EN 1993-1-1 [1].

\subsubsection{Stiffness reduction function for bending $\tau_{M}$}

The stiffness reduction function for bending $\tau_{M}$ adopted in Kucukler et al. [13] is expressed as follows:

$$
\begin{array}{r}
\tau_{M}=1.0 \quad \text { if } \quad C_{m} M_{y, E d} / M_{y, p l} \leq \phi_{y}, \\
\tau_{M}=\left(1-\tau_{M l}\right)\left[1-\left(\frac{C_{m} M_{y, E d} / M_{y, p l}-\phi_{y}}{\xi_{m}-\phi_{y}}\right)^{\beta_{m}}\right]^{1 / \beta_{m}}+\tau_{M l} \quad \text { if } \quad \phi_{y} \leq C_{m} M_{y, E d} / M_{y, p l} \leq \xi_{m}, \\
\tau_{M}=\tau_{M l}\left[1-\left(\frac{C_{m} M_{y, E d} / M_{y, p l}-\xi_{m}}{1-\xi_{m}}\right)\right]^{1 / \delta_{m}} \quad \text { if } \quad \xi_{m} \leq C_{m} M_{y, E d} / M_{y, p l},
\end{array}
$$

where $M_{y, E d}$ is the maximum absolute bending moment value along the length of the steel member, $M_{y, p l}$ is the major axis plastic bending moment resistance equal to the plastic section modulus about the major axis $W_{p l, y}$ multiplied by the yield stress (i.e. $M_{y, p l}=$ $\left.W_{p l, y} f_{y}\right)$ and $\tau_{M l}, \beta_{m}, \phi_{y}, \delta_{m}$ and $\xi_{m}$ are the auxiliary coefficients whose values are provided in Table 2 for wide and narrow flange sections, as recommended by Zubydan [7]. In the stiffness reduction function for bending $\tau_{M}$ given by eq. (2), the maximum bending moment 
value along the length is multiplied with a moment gradient factor $C_{m}$ to consider the influence of bending moment shape on the development of plasticity. The moment gradient factor $C_{m}$ can be determined using the following expression [21]:

$$
C_{m}=\frac{-1.5 M_{y, E d}+4 M_{A}+6 M_{B}+4 M_{C}}{12.5 M_{y, E d}}
$$

in which $M_{A}, M_{B}$ and $M_{C}$ are the absolute values of bending moments at the quarter, centre and three-quarter points of the length of the steel member and $M_{y, E d}$ is the maximum absolute bending moment value along the length. It should be noted that the expression for the moment gradient factor $C_{m}$ given by eq. (3) was developed through calibration to the GMNIA results of simply-supported beam-columns, and gives improved accuracy in comparison to the adoption of the expression of Austin [22] or that of Kirby and Nethercot [23], which was previously recommended by Kucukler et al. [13].

\subsubsection{Stiffness reduction function for combined axial loading and bending $\tau_{M N}$}

The stiffness reduction function for combined axial loading and bending $\tau_{M N}$ proposed by Kucukler et al. [13] is given by the following equation:

$$
\tau_{M N}=\tau_{M} \tau_{N}\left\{1-\left(\frac{N_{E d}}{N_{p l}}\right)^{0.8}\left(\frac{C_{m} M_{y, E d}}{M_{y, p l}}\right)\right\} .
$$

Similar to $\tau_{M}$, the moment gradient factor $C_{m}$ determined using eq. (3) is multiplied with the maximum absolute bending moment value along the member length $M_{y, E d}$ in $\tau_{M N}$ to consider the influence of bending moment shape on the development of plasticity. $\tau_{M N}$ degenerates into $\tau_{N}$ for pure axial compression and $\tau_{M}$ for pure bending. The stiffness reduction function $\tau_{M N}$ is employed to reduce the flexural stiffness of steel members whereby the detrimental influence of residual stresses, geometrical imperfections and the combined effect of bending moment and axial loading on the spread of plasticity are fully taken into account.

It is worth noting that since this paper investigates the application of the proposed approach to frames with regular, prismatic steel members, the influence of bending moment shape on the plasticity is conveniently taken into account by incorporating moment gradient factors into the stiffness reduction functions. In the case of a frame involving irregular members such as tapered or stepped beam-columns or curved members, the tapering approach, where the member is divided into portions along its length and the flexural stiffnesses of each portion are reduced by considering the corresponding bending moments through $\tau_{M N}$ with $C_{m}=1.0$ as described in Kucukler et al. [14, 15], can be used.

\subsection{Ultimate cross-section strength equations}

Owing to its simple form and high accuracy, the ultimate cross-section strength equation proposed by Duan and Chen [24] was used in this study. The ultimate cross-section strength equation of [24] for major axis bending $M_{y, E d}$ plus axial loading $N_{E d}$ is given by:

$$
1 / \alpha_{u l t, c}=\left(\frac{N_{E d}}{N_{p l}}\right)^{1.3}+\frac{M_{y, E d}}{M_{y, p l}} \leq 1,
$$


where $1 / \alpha_{u l t, c}$ is the cross-section utilisation factor. In cases of high shear, $N_{p l}$ and $M_{y, p l}$ should be reduced following the recommendations given in Clause 6.2.10 of EN 1993-1-1 [1].

\subsection{Application of the stiffness reduction method}

The proposed stiffness reduction method (SRM) is applied to the investigated frames by adopting the following steps in this paper:

(i) Perform Linear Elastic Analysis (i.e. first-order elastic analysis) considering the out-ofplumbness of the frame equal to the maximum value $0.002 \mathrm{rad}$ allowed by EN 1090-2 $[25]$.

(ii) Calculate stiffness reduction factors $\tau_{M N}$, whose expression is given by eq. (4), using the forces $N_{E d}$ and $M_{y, E d}$ determined through Linear Elastic Analysis for each member and reduce their flexural stiffnesses.

(iii) Perform Geometrically Nonlinear Analysis with reduced stiffness (GNA-SR), taking into account the out-of-plumbness of the frame equal to 0.002 rad.

(iv) Make cross-section checks using the internal member forces obtained through GNA-SR employing eq. (5), where the cross-section utilisation factor $1 / \alpha_{u l t, c}$ must be equal to or less than 1.0 (i.e. $1 / \alpha_{u l t, c} \leq 1.0$ ) within the frame.

An important point that should be highlighted is that the SRM must be implemented through software able to perform Geometrically Nonlinear Analysis (GNA) taking into account fully both $P-\delta$ and $P-\Delta$ effects. GNA enables the consideration of both member and frame instabilities, the influence of boundary conditions and restraint of adjoining members, while load level and the corresponding influence of geometrical imperfections, residual stresses and the spread of plasticity are allowed for through stiffness reduction.

The influence of frame out-of-plumbness may be considered by direct modelling or applying notional horizontal forces to the frame with perfect geometry in the proposed SRM. In the latter case, the magnitude of the notional horizontal force applied to a storey should be equal to 0.002 times of the total factored gravity load applied at that storey. The application of the notional loads to the frames for the representation of the initial out-of-plumbness is described in detail in AISC 360-10 [2] and EN 1993-1-1 [1] and the same principles apply to the SRM presented in this paper.

It should be noted that in the case of a frame involving a member subjected to pure compression (with zero bending moment), a small horizontal force at the the mid-height of the member should be applied in GNA-SR to generate a perturbation resulting in its flexural buckling.

Finally, it is worth emphasising that in the SRM, the flexural stiffnesses $(E I)$ of the members need to be reduced through $\tau_{M N}$ along the whole member length, which should be achieved by reducing their second moments of area $I$, since reducing the Young's modulus $E$ may also lead to unintended differential reductions in the axial stiffnesses $(E A)$ of the members, which may result in artificial distortion of the structure [2]. 


\section{Application of the stiffness reduction method to simply-supported beam- columns under varying bending moments}

In this section, the accuracy of the proposed stiffness reduction method (SRM) is investigated for simply-supported steel beam-columns subjected to 42 loading cases shown in Table 3 for major axis bending and axial compression. The 42 considered loading cases correspond to various bending moment shapes observed in steel structures. The SRM was applied by adopting the steps described in Section 3.3 with the exception of the consideration of outof-plumbness. Three cross-section shapes were considered: IPE500, HEB400 and HEB200 representing the response of beam-columns with cross-sections having large, average and small depth-to-width ratios respectively. For each loading combination shown in Table 3 , the non-dimensional slendernesses of the analysed beam-columns were $\bar{\lambda}_{y}=0.4, \bar{\lambda}_{y}=1.0$ and $\bar{\lambda}_{y}=1.5$, enabling verification of the SRM for beam-columns with low, moderate and high slendernesses. Note that $\bar{\lambda}_{y}$ was determined by taking the square root of the ratio of the axial yield load $N_{p l}$ to the major axis elastic flexural buckling load $N_{c r, y}$ of the member (i.e. $\bar{\lambda}_{y}=\sqrt{N_{p l} / N_{c r, y}}$ ). The accuracy of the SRM was assessed through the parameter $\epsilon$, which was determined using the following equation:

$$
\begin{array}{r}
\epsilon=\frac{R_{S R M}}{R_{G M N I A}}=\frac{\sqrt{\left(N_{E d, \max , S R M} / N_{p l}\right)^{2}+\left(M_{y, E d, \max , S R M} / M_{y, p l}\right)^{2}}}{\sqrt{\left(N_{E d, \max , G M N I A} / N_{p l}\right)^{2}+\left(M_{y, E d, \max , \text { GMNIA }} / M_{y, p l}\right)^{2}}} \\
\text { but } \frac{M_{y, E d, \max , S R M}}{N_{E d, \max , S R M}}=\frac{M_{y, E d, \max , G M N I A}}{N_{E d, \max , \text { GMNIA }}},
\end{array}
$$

where $R_{S R M}$ and $R_{G M N I A}$ are the radial distances measured from the origin to the ultimate strength $M_{y, E d}-N_{E d}$ interaction curves determined through the SRM and GMNIA respectively. In eq. (6), $N_{E d, \max , S R M}$ and $M_{y, E d, \max , S R M}$ are the ultimate axial compression and bending strengths obtained through the SRM and $N_{E d, \max , G M N I A}$ and $M_{y, E d, \max , \text { GMNIA }}$ are the ultimate axial compression and bending strengths obtained through GMNIA respectively. As can be interpreted from eq. (6), $\epsilon$ values larger than 1.0 correspond to unconservative strength predictions.

The comparison of the strength predictions made by the SRM against those obtained through GMNIA is presented in Table 4, where, in total, 2997 simply-supported beamcolumns are considered. In Table $4, \mathrm{~N}$ is the number of beam-columns analysed for a particular group, $\epsilon_{a v}$ and $\epsilon_{c o v}$ are the average and coefficient of variation of $\epsilon$ values respectively, and $\epsilon_{\max }$ and $\epsilon_{\min }$ are the maximum and minimum values of $\epsilon$ in the considered range. Note that for each loading condition and slenderness value, $\epsilon$ values were determined considering 10 different points within the $M_{y, E d}-N_{E d}$ interaction curve, which corresponded to different ratios of bending moment to axial compression. In the cases where GMNIA interaction curves exceeded the ultimate cross-section strength surface due to strain hardening, $\epsilon$ values were not calculated. As can be seen from Table 4, the SRM provides quite accurate ultimate strength predictions where $\epsilon_{a v}$ values are very close to 1.0 with small coefficient of variation $\epsilon_{\text {cov }}$ values for various loading conditions, different slenderness values and different ratios of bending moment to axial compression. In the investigated wide range of cases, the SRM 
provides unconservative errors no greater than $6 \%$ (i.e. $\epsilon \leq 1.06$ ), indicating the reliability of the proposed SRM.

To assess the accuracy of the proposed SRM in comparison to other structural steel design approaches, the ultimate strengths predicted by the direct analysis method (DAM) of AISC 360-10 [2] and the beam-column design methods given in the Annexes A and B of EN 1993-1-1 [1] are also compared against those of GMNIA in Table 4. Note that the $\epsilon$ values were calculated by taking the ratios of the radial distances measured from the origin to the ultimate strength $M_{y, E d}-N_{E d}$ interaction curves obtained through the corresponding design method and GMNIA respectively. Since a safety factor, equal to 0.9 and taking into account the uncertainties with respect to material properties and cross-section dimensions, was not applied to strength and stiffness in the GMNIA simulations, the stiffness reduction factor equal to 0.8 , which also considers these uncertainties, was modified by taking $0.8 / 0.9 \approx 0.9$ in the application of the DAM. Thus, in lieu of $0.8 \tau_{b}$, the stiffness reduction expression corresponding to $0.9 \tau_{b}$ was used and the member strengths were not multiplied by 0.9 in the application of the DAM in this paper, which is in accordance with the recommendations of Surovek-Maleck and White [11]. Note that $\tau_{b}$ is the stiffness reduction function of the DAM applied to members with $N_{E d} / N_{p l}>0.5$, whose expression can be found in AISC 360-10 [2]. Table 4 shows that the DAM generally provides overly conservative ultimate strength predictions and the proposed SRM leads to more accurate results in all the considered cases. This results from the fact that while the stiffness reduction functions of the SRM are able to consider the influence of bending moment distribution on the development of plasticity, the DAM uses a constant stiffness reduction factor to account for plasticity associated with bending regardless of the shape of the bending moment diagram. It is worth noting that while the same geometrical imperfection shape and magnitudes used in this paper were assumed in the development of the DAM [11], the adopted residual stress pattern was different than those assumed in this paper, which may have some influence on the results presented in this and following sections.

Table 4 also shows the comparison between the ultimate strengths obtained through the proposed SRM and those obtained through the beam-column design methods presented in Eurocode 3 [1] Annex A (EC3-A) and Annex B (EC3-B), where it is seen that the SRM leads to more accurate ultimate strength predictions in all the considered cases, though the Eurocode 3 [1] beam-column design methods are more accurate than the DAM of AISC 360-10 [2]. It is noteworthy that the adoption of the more precise nonlinear cross-section strength interaction equation of Duan and Chen [24] by the SRM in comparison to the interaction equations provided in AISC 360-10 [2] and EN 1993-1-1 [1] partly contributes to the higher accuracy of the SRM observed in this and following sections.

\section{Application of the stiffness reduction method to frames with low redundancy}

The application of the SRM to frames with low redundancy is addressed in this section. Two examples are taken into consideration: sway-inhibited and sway-uninhibited single-storey, single-span frames. In all cases, the ultimate capacities and the internal forces of the frames are compared against those obtained through GMNIA. Moreover, the pro- 
posed method is also compared against the direct analysis method (DAM) and notional load method (NLM) of Eurocode 3 [1]. In the implementation of the SRM, DAM and NLM, the Geometrically Nonlinear Analyses (GNA) of the frames were performed through a Matlab [26] code based on the stability function approach recommended by Chen and Toma [27], using sixteen beam-column elements to model each member of the non-sway frames and four beam-column elements to model each member of the sway frames.

\subsection{Rigid-jointed non-sway frames}

This subsection presents the assessment of the accuracy of the SRM for the sway-inhibited rigid-jointed steel frame shown in Fig. 3. The studied frame is similar to that investigated by Liew et al. [5] but a broader range of geometrical properties and section sizes are considered herein. As can be seen from Fig. 3, the pinned based frame is subjected to concentrated forces $N_{E d}$ at the top of the columns and a uniformly distributed load $w$ along the beam. In the figure, $I_{b}$ and $I_{c}$ are the second moments of area about the major axis of the beam and columns respectively. Different magnitudes of concentrated loads $N_{E d}$ and uniformly distributed load $w$ were applied to the frames considering $\beta=w 3 L /\left(w 3 L+2 N_{E d}\right)$, where $\beta$ was varied between 0 and 1 so as to assess the accuracy of the SRM for the prediction of the bending moment values transferred from the beam to the columns in the cases where the columns are subjected to various levels of axial compression. In the frames, the beam and columns were orientated such that they were under major axis bending. Since the proposed SRM requires the application of different rates of stiffness reduction to members within a frame on the basis of the first-order forces they withstand, it leads to internal forces different than those calculated through elastic analysis with nominal stiffnesses. Since the analysed frame is not susceptible to any $P-\Delta$ effects, it is a sensitive benchmark for the assessment of the accuracy of the SRM with respect to the prediction of internal forces within steel frames, which is strongly influenced by the change in the flexural stiffnesses of their members.

As illustrated in Table 5, to assess the importance of different parameters, two crosssection shapes, HEB400 and HEB200, with different residual stress patterns, as shown in Fig. 2, were considered for the columns. Moreover, the cross-section shapes of the beams were also varied to obtain different ratios of the flexural stiffnesses of the columns to those of the beams, as represented by the parameter $G_{R}=\left(E I_{c} / L\right) /\left(E I_{b} / 3 L\right)$. As can be seen from Table 5 , the beam cross-sections were chosen such that $G_{R}$ values were equal to $0.35-0.36$, 1.01-1.07 and 3.00, representing strong beam-weak column, equally strong beam and column and weak beam-strong column cases respectively. Table 5 also shows that the plastic bending moment resistance of the columns $M_{y, p l, c}$ were generally lower than those of the beams $M_{y, p l, b}$.

In the implementation of the SRM, the DAM and the GMNIA simulations, the frames were loaded proportionally such that the applied loading led to the attainment of the ultimate cross-section strength at the top of the columns or at the ends of the beams according to the Linear Elastic Analysis (LA) on the basis of the cross-section strength equation given by eq. (5). This type of loading of the frames led to the largest differences with respect to the rates of stiffness reduction applied to the columns and the beams according to the proposed SRM, where the reduction rates of the stiffness of the columns were much larger. In the calculations, nine $\beta$ values equal to $\beta=1.0,0.8,0.6,0.4,0.3,0.2,0.1,0.05,0.02$ were 
used. For each $\beta$ and $G_{R}$ value and column cross-section shape, non-dimensional slenderness $\bar{\lambda}_{y}$ values equal to $0.4,1.0$ and 1.5 (i.e. $\bar{\lambda}_{y}=0.4,1.0,1.5$ ) were considered for the columns, thus assessing the response of sway inhibited frames having columns with low, moderate and high slenderness. Note that $\bar{\lambda}_{y}=\sqrt{N_{p l} / N_{c r, y}}$ values were determined using $N_{c r, y}$ values calculated considering the actual lengths of the columns.

The comparison of the internal forces determined through the SRM and the DAM against those of GMNIA is presented in Table 6 , in which $\mathrm{N}$ is the number of the investigated frame cases for each group. The accuracy of the internal force prediction was assessed considering the $\zeta$ parameter, whose expression is given by eq. (7):

$$
\zeta=\frac{\sqrt{\left(N_{E d, p r e d i c t} / N_{p l}\right)^{2}+\left(M_{y, E d, p r e d i c t} / M_{y, p l}\right)^{2}}}{\sqrt{\left(N_{E d, G M N I A} / N_{p l}\right)^{2}+\left(M_{y, E d, G M N I A} / M_{y, p l}\right)^{2}}},
$$

where $N_{E d, p r e d i c t}$ and $M_{y, E d \text {,predict }}$ are the axial force and bending moment at the top of the columns predicted by the corresponding design method and $N_{E d, G M N I A}$ and $M_{y, E d, G M N I A}$ are the axial force and bending moment at the top of the columns determined by the GMNIA of a frame. The quantities $\zeta_{a v}$ and $\zeta_{c o v}$ are the average and coefficient of variation of the $\zeta$ values, and $\zeta_{\max }$ and $\zeta_{\min }$ are the maximum and minimum of values of $\zeta$; $\zeta$ values less than 1.0 indicate that the bending moment transferred from the beam to the columns is underpredicted. Note that in a few GMNIA simulations, the frames failed before attaining the load factor leading to the exploitation of the cross-section resistance at the top of the columns according to the Linear Elastic Analysis. In these cases, $\zeta$ values were not calculated. The $\epsilon_{\max }$ values which correspond to the maximum values of the ratios of the ultimate load carrying capacities determined by the SRM or the DAM to those determined through GMNIA (i.e. $\left.\epsilon_{\max }=\max \left(\alpha_{u l t} / \alpha_{u l t, G M N I A}\right)\right)$ are also provided in Table 6 .

Table 6 shows that the SRM generally underestimates the bending moments transferred from the beam to the columns. The rate of this underprediction is inversely related to the $G_{R}$ values (i.e. larger underpredictions were observed for strong beam-weak column cases). The underprediction of the bending moments within the columns results from the application of significantly higher rates of stiffness reduction to the columns in comparison to the beams from the onset of the analysis (i.e. GNA-SR), though in reality the stiffness of the columns degrade gradually and the bending moments transferred from the beam to the columns start to reduce in the later stages of the loading history. The results obtained through the DAM are also compared against those of GMNIA in Table 6, where it is seen that the method leads to accurate predictions of internal forces within the columns. It is worth noting that some fraction of the discrepancies between the internal force predictions shown in Table 6 may result from the neglect of the shear deformations in the SRM and DAM, while they were taken into account in the GMNIA. Nevertheless, this is expected to be of minor influence on the assessment of the accuracy of the internal force predictions carried out in this subsection.

The underestimation of the bending moments within the columns by the SRM does not lead to significant overpredictions of the in-plane strengths of the frames as the $\epsilon_{\max }$ values are less than or equal to 1.06 in all the considered frame cases which can be seen from Table 6. Nevertheless, for frames susceptible to flexural-torsional buckling effects, this may lead to 
significant overpredictions of the strengths. To reduce the extent of the underprediction of the bending moments within the columns of steel frames, this study recommends the use of an upper limit to the stiffness reduction factor $\tau_{M N, l i m}$, where the stiffness reduction factors applied to all the members of a frame, including the beams, should be less than or equal to $\tau_{M N, l i m}$. The expression of the upper limit to the stiffness reduction factor $\tau_{M N, l i m}$ is given by:

$$
\tau_{M N, \text { lim }}=\max \left\{\tau_{M N, \min } ; 0.8\right\},
$$

which has been obtained through the calibration to the internal forces determined through GMNIA for sway-inhibited rigid jointed frames considered in this subsection. As can be seen from the equation, the limit stiffness reduction factor $\tau_{M N, l i m}$ is equal to the larger of the minimum of the stiffness reduction factors determined for all members of a frame which attract bending moment from neighbouring members $\tau_{M N, \min }$ or 0.8 . For instance, if the minimum of the stiffness reduction factors $\tau_{M N, \min }$ calculated for all the members attracting bending moment is equal to 0.6 , the members should have stiffness reduction factors less than or equal to 0.8 (i.e. the $\tau_{M N}$ for all the members should not exceed $\tau_{M N, l i m}=0.8$ ). On the other hand, if the minimum of the stiffness reduction factors is equal to 0.9, all the members attracting bending moments should have the same stiffness reduction factor equal to 0.9 (i.e. $\tau_{M N, l i m}=0.9$ ). Thus, according to eq. (8), the same stiffness reduction factor equal to $\tau_{M N, \text { lim }}=\tau_{M N \text {, min }}$ should be applied to all the members in the cases where $\tau_{M N, \min }$ is larger than or equal to 0.8. The purpose of imposing an upper bound to the stiffness reduction factor is to reduce the differences between the stiffness reduction factors applied to the beams and columns, thereby achieving better internal force predictions for moment-resisting frames.

The comparison of the internal forces and ultimate strength values predicted by the proposed stiffness reduction method with $\tau_{M N, l i m}$ (i.e. SRM-Limit) against those of GMNIA is presented in Table 6. As can be seen from the table, the proposed modification results in significant improvements with respect to the predictions of the internal forces within the columns, as generally higher rates of reduction are applied to the flexural stiffness of the beams through $\tau_{M N, l i m}$. SRM-Limit leads to $\zeta_{a v}$ values very close to 1.0 with small coefficient of variation $\zeta_{\text {cov }}$ values. It should be borne in mind that the frames considered in this subsection represent the most unfavourable cases where the loading conditions result in the attainment of the ultimate cross-section resistance within the columns according to the Linear Elastic Analysis, thus leading to the largest possible discrepancies with respect to the rates of stiffness reduction applied to the columns and the beams. In the case of any outof-plane instability effects, the applied forces will be smaller due to the reduced resistance of the structure, which will lead to the application of lower rates of stiffness reduction (i.e. larger values of $\tau_{M N}$ ) to the columns, thus resulting in larger rates of moment transfer to these members. The same case also applies to sway-uninhibited frames whose resistance is reduced due to $P-\Delta$ effects, which will also increase second-order bending moments within the columns.

Based on the findings of this subsection, this paper recommends the application of the proposed SRM with the upper limit stiffness reduction factor assumption, where the upper 
limit stiffness reduction factor $\tau_{M N, l i m}$ is determined using eq. (8). This type of application of the SRM will be adopted in the following sections of this paper. It should be emphasised that the upper limit stiffness reduction factor concept is concerned with the members of a frame attracting bending moments from neighbouring members. Thus, if a frame has leaning columns (i.e. columns pinned at both ends), $\tau_{M N, l i m}$ should be determined excluding the $\tau_{M N}$ values for the leaning columns and should not be applied to these members. In the case of pin-jointed braced frames (i.e. simple construction), of course, the proposed stiffness reduction approach should be applied without the upper limit stiffness reduction factor assumption.

\subsection{Kanchanalai [28] non-redundant sway frames}

In this subsection, the application of the SRM to the non-redundant frames originally studied by Kanchanalai [28] whose geometrical properties and loading conditions are shown in Fig. 4 is investigated. As can be seen from Fig. 4, four different frame configurations, subjected to horizontal loading $H_{E d}$ plus vertical loads $N_{E d}$ applied at the top of the columns, are considered. While the frames shown in Figs. 4 (a) and (b) involve both columns contributing to the lateral stability, those illustrated in Figs. 4 (c) and (d) have leaning columns which are pinned at both ends, thus not providing any lateral stability to the structure. The presence of a leaning column within the frame generates additional second-order forces within the column providing the lateral stability, which may significantly influence the behaviour. To assess the capability of the SRM for the consideration of the leaning column effects, the vertical loads applied to the leaning columns $\gamma_{L} N_{E d}$ were varied such that the $\gamma_{L}$ values, representing the ratio of the vertical load applied to the leaning column to that applied to the column providing the lateral stability, were equal to 1.0, 2.0 and 3.0.

The Kanchanalai [28] frames are labelled herein considering their base support conditions and the presence of leaning columns within them. The FP frame is a pinned based frame, the LFP frame is a pinned based frame but also involves a leaning column, the FR frame is a frame with a base beam providing rotational restraint at the supports, and the LFR frame is a frame with a base beam and a leaning column. In the frames, columns with HEB 400 and HEB 200 cross-sections were used so as to investigate the accuracy of the SRM for frames with columns with narrow flange (i.e. $h / b>1.2$ ) and wide flange (i.e. $h / b \leq 1.2)$ cross-sections, corresponding to different residual stress patterns, as shown in Fig. 2. In line with [28] and [12], the span to height ratios of the frames were assumed to be very large (i.e. $L_{b} \gg L$ ), thus the overturning effects due to the horizontal loading $H_{E d}$ were insignificant. The beams and columns in the frames were under major axis bending. For the case of the FP and LFP frames, three slenderness values $\lambda_{y}$ equal to 20, 40,60, corresponding to the ratios of the lengths of the columns to their radii of gyration about the major axis $i_{y}$ (i.e. $\lambda_{y}=L / i_{y}=20,40,60$ ), were considered. For the FR and LFR frames, $\lambda_{y}=L / i_{y}=40,60,80$ were used. The second moments of area of the beams $I_{b}$ were specified such that the $G_{R}$ parameters representing the ratio of the flexural stiffness of the columns $\left(E I_{c}\right)$ to that of the beams (i.e. $\left.G_{R}=\left(I_{c} / L\right) /\left(I_{b} / L_{b}\right)\right)$ were equal to 0,1 and 3 for the FP and FR frames and to 0 and 2 for the LFP and LFR frames. The same values for the second moments of area were used for the base and top beams in the FR and LFR frames. 
The comparison of the ultimate strengths predicted through the SRM against those obtained from GMNIA and the DAM is presented in Fig. 5, where $N_{p l}$ and $M_{y, p l}$ correspond to the axial yield load and major axis plastic bending moment resistance of the columns respectively. As can be seen from the figure, the SRM provides accurate strength predictions for the Kanchanalai [28] frames for different column-to-beam stiffness ratios $G_{R}$, base support conditions, column slenderness $\lambda_{y}$ values and leaning column effects $\gamma_{L}$. Fig. 5 also shows that the ultimate strengths determined through the DAM are generally in good agreement with those of GMNIA with the exception of the FR frames. In the case of the FR frames, where the columns are subjected to double curvature bending, the SRM leads to more accurate predictions of the strengths in comparison to the DAM owing to the use of moment gradient factors $C_{m}$ within its stiffness reduction functions $\tau_{M N}$ as can be seen from Fig. 5 (b). Nevertheless, the comparison of Fig. 5 (b) and Fig. 5 (d) indicate that the use of the moment gradient factors $C_{m}$ within the stiffness reduction functions becomes of less importance for frames with leaning columns, despite the columns providing the lateral stability being under double curvature bending.

In addition to the proposed SRM and the DAM, the ultimate strengths determined through the notional load method (NLM) of Eurocode 3 [1] are also compared against those obtained through GMNIA in Fig. 5. The NLM of Eurocode 3 [1] utilises an equivalent outof-plumbness value, which is larger than the maximum out-of-plumbness value permitted by the corresponding European standard with respect to the fabrication and erection of steel structures [25], to consider both the influence of the spread of plasticity and the actual outof-plumbness on the response of a steel structure. Since the influence of equivalent frame out-of-plumbness is usually represented through notional horizontal loads, this approach is referred to as the notional load method (NLM) in the literature [29]; this definition is also adopted in this paper. Note that in the case of the NLM, the ultimate strength $H_{E d}-N_{E d}$ interaction curves determined through both the beam-column design methods provided in Eurocode 3 [1] Annex A (NLM EC3-A) and Annex B (NLM EC3-B) are presented. Following the recommendations of Boissonnade et al. [30], exact equivalent moment factors determined by performing Geometrically Nonlinear Analyses of the frames were used in the calculations. In the application of the NLM in this and following sections, the base value of the equivalent out-of-plumbness of 0.005 rad is employed, since the actual out-of-plumbness of the frames is not varied considering their heights and the number of columns within a storey in the GMNIA simulations. Fig. 5 shows that both the NLM EC3-A and NLM EC3-B lead to overly conservative strength estimations and the SRM provides considerably more accurate results particularly for the FR and LFR frames. The drop in accuracy of the Eurocode 3 [1] beam-column design methods observed herein is in accordance with the statements of Boissonnade et al. [30] indicating that the design methods were originally developed for simply-supported beam-columns and a loss of accuracy is expected for other cases.

The accuracy of the SRM, DAM and NLM EC3-A and NLM EC3-B was also investigated for an additional $817 \mathrm{FP}, \mathrm{FR}, \mathrm{LFP}$ and LFR frames with different $G_{R}$ and $\gamma_{L}$ values, as summarised in Table 7, where $\mathrm{N}$ is the number of frames considered for each group. Note that HEB 200 and HEB 400 sections were used within the columns with $\lambda_{y}=L / i_{y}=20,40,60$ in the case of the FP and LFP frames and with $\lambda_{y}=L / i_{y}=40,60,80$ in the case of the 
FR and LFR frames. Following the approach adopted in Section 4, the accuracy of the SRM was assessed using the $\epsilon$ parameter, which is the ratio between $R_{S R M}$ and $R_{G M N I A}$ (i.e. $\epsilon=R_{S R M} / R_{G M N I A}$ ), where $R_{S R M}$ and $R_{G M N I A}$ are the radial distances measured from the origin to the $N_{E d}-H_{E d}$ interaction curves determined through SRM and GMNIA, respectively. Note that these distances were normalised by $N_{E d} / N_{p l}$ and $M_{y, E d} / M_{y, p l}$, where $M_{y, E d}$ is the maximum first-order bending moment within the column generated by $H_{E d}$; $\epsilon$ values greater than 1.0 indicate unconservative strength predictions. In the case of the DAM, NLM EC3-A and NLM EC3-B, the $\epsilon$ values were calculated by taking the ratios of the radial line distances from the origin to the interaction curves determined by the corresponding design method and GMNIA respectively.

Table 7 shows that the SRM provides accurate and safe ultimate strength predictions for the Kanchanalai [28] frames, though its accuracy decreases for the high $G_{R}$ values. This results from the adoption of the upper bound stiffness reduction factor assumption introduced in the previous subsection, where the flexural stiffness of the beams are reduced using a factor $\tau_{M N, \text { lim }}$ given by eq. (8), though their actual stiffness reduction factors are very close to 1.0, which significantly increases the $P-\Delta$ effects in some cases. Nevertheless, the SRM is still more accurate than the NLM EC3-A and NLM EC3-B and provides unconservative results of no greater than $3 \%$ (i.e. $\epsilon_{\max }=1.03$ ) even for these non-redundant 817 frames highly sensitive to the spread of plasticity and instability effects. The comparison of the SRM and DAM indicates that the DAM generally provides more accurate results than the SRM due to the use of lower rates of stiffness reduction for the beams in the former. Yet the SRM leads to more accurate results for the majority of the FR frames and the differences between the strength predictions are not significant for the other frames.

\section{Application of the stiffness reduction method to frames with high redundancy}

In this section, the application of the SRM to a series of redundant benchmark frames from the literature is illustrated. The NLM of Eurocode 3 [1] and the DAM of AISC-360-10 [2] are also applied to the considered frames so as to compare the SRM against these structural steel design approaches. The ultimate strength values and internal force predictions obtained through the SRM, DAM and NLM are compared against those of GMNIA in all the considered cases. In the case of the DAM and NLM, the ultimate strength values for the frame were determined using the load factors at which any member first violated the beamcolumn interaction equations of AISC 360-10 [2] and EN 1993-1-1 [1] respectively. Unlike the previous sections, shear deformations are accounted for in the implementation of the SRM, DAM and NLM in this section so as to eliminate any minor discrepancies resulting from different analysis assumptions (i.e. Euler-Bernoulli or Timoshenko beam theories) in the assessment of the internal force predictions. In the implementation of the SRM, DAM and NLM, the Geometrically Nonlinear Analyses were performed through the MASTAN2 [31] structural analysis software using 4 elements to model each individual member of the frames. In all the GMNIA, SRM, DAM and NLM, the fillets of the cross-sections were not taken into account and the frames were loaded proportionally. 


\subsection{Application of the stiffness reduction method to Ziemian [32] frames}

This subsection investigates the application of the SRM to two-storey moment resistant frames whose geometrical properties and loading conditions are shown in Fig. 6. As shown in Fig. 6, the frames which were originally studied by Ziemian [32] are symmetrical or unsymmetrical, subjected to heavy $(\mathrm{H})$ or light $(\mathrm{L})$ gravity loading, pinned or fixed based and are made up of steel whose yield stress is $248 \mathrm{MPa}$ (i.e. $36 \mathrm{ksi}$ ) or $345 \mathrm{MPa}$ (i.e. $50 \mathrm{ksi}$ ). Considering the combination of these parameters in line with [32], in total, 16 frames are investigated herein. Note that both heavy and light gravity loads involve equal portions of dead $\left(G_{D}\right)$ and live $(Q)$ loads, and the wind $(W)$ load is the same for all the frames. The section sizes of these frames are assumed to be the same as those determined on the basis of the elastic LRFD [33] design by Ziemian [32]. All the section sizes of the frames can be found in Ziemian [32]. As indicated by [32], the gravity loading combination governs the strengths of the frames. Thus, only one load combination corresponding to the largest gravity loading is considered herein in accordance with EN 1990 [34]: 1.35G $G_{D}+1.5 Q+0.75 \mathrm{~W}$.

The comparison of the results is presented in Table 8. The frames are named according their properties in that $\mathrm{U}$ and $\mathrm{S}$ indicate that the frame is unsymmetric or symmetric respectively, $\mathrm{P}$ and $\mathrm{F}$ show that the frame is fixed or pinned based, 36 and 50 represent the yield stress of the steel used in the frame is $248 \mathrm{MPa}$ (i.e. $36 \mathrm{ksi}$ ) or $345 \mathrm{MPa}$ (i.e. 50 $\mathrm{ksi}$ ), and $\mathrm{L}$ and $\mathrm{H}$ show whether the frame is subjected to light or heavy gravity loading respectively. Thus, for instance, UP36H means an unsymmetric pinned based frame with steel whose yield strength is $248 \mathrm{MPa}$ and subjected to heavy gravity loading. In Table 8 , the elastic critical buckling load amplifiers $\alpha_{c r}$ of the frames associated with the global sway buckling modes are also given so as to provide an indication of their sensitivity to the second-order effects. The accuracy of the different design methods for the prediction of the internal forces is assessed considering the $\zeta$ parameter whose expression is given by eq. (7), where $N_{E d, p r e d i c t}$ and $M_{y, E d, p r e d i c t}$ are the axial force and maximum second-order bending moment within a member predicted by a design method, and $N_{E d, G M N I A}$ and $M_{y, E d, G M N I A}$ are the axial force and maximum second-order bending moment within a member predicted by GMNIA. Thus, a $\zeta$ value smaller than 1.0 indicates that the internal forces within the member are underpredicted. In Table $8, \zeta_{a v}$ and $\zeta_{\text {cov }}$ are the average and coefficient of variation, with $\zeta_{\max }$ and $\zeta_{\min }$ being the maximum and minimum values of $\zeta$ determined for each member of a frame. The ultimate load amplifier $\alpha_{u l t}$ of the frames determined through the different methods are also provided in Table 8.

As can be seen from Table 8, the SRM leads to safe and accurate internal force predictions for the 16 Ziemian [32] frames with symmetric and unsymmetric geometries, base support conditions, material properties and loading conditions. Moreover, the SRM provides values of the internal forces and capacities quite close to those determined through the DAM for the majority of the frames. It should be noted that in the case of the unsymmetric frames, the wind loads were applied in both directions and the largest forces influencing each member were taken into account in the calculation of the $\zeta$ values in Table 8 . In the case of the symmetric frames, the $\zeta$ values were determined considering the internal forces within the critical leeward columns and windward beams and the middle columns. The NLM also provides strength predictions, calculated using the Eurocode 3 [1] Annex A beam-column 
design method, close to the SRM and DAM but the estimated internal forces are somewhat more conservative as the $\zeta_{a v}$ values are between 1.02 and 1.04. The discrepancies between the internal forces determined through the different design methods and those of GMNIA primarily result from the different predictions of the bending moments within the members; the predictions of the axial forces are generally very close. Although both the SRM and DAM underpredict the internal forces for some members, these members are not critical and generally subjected to small internal forces. Moreover, $\zeta_{a v}$ values determined through both the SRM and DAM are generally close to 1.0 with small $\zeta_{\text {cov }}$ values, which indicate the accuracy of the methods with respect to internal force predictions. The SRM provides rather conservative internal force predictions for the slender frames (i.e. the frames with $\alpha_{c r} \leq 3.0$ ), which may result from the application of the upper bound stiffness reduction factor $\tau_{M N, l i m}$ to the lightly loaded members. The internal forces predicted through the SRM are close to those determined through the NLM though and the overprediction of the internal forces does not have marked influence on the capacity predictions for these slender frames.

Since the SRM, DAM and NLM approaches are all elastic analysis and design methods where the failure is signified by the development of the first plastic hinge, they generally provide quite conservative strength predictions $\alpha_{u l t}$ in comparison to GMNIA for the Ziemian [32] frames. This results from the fact that these frames have high reserves of plastic strengths, generally failing in the sway mode with the development of multiple plastic hinges as shown by Ziemian et al. [19]. It is also worth noting that since the frames considered in this section are generally well proportioned, the ultimate strength predictions obtained through the SRM, DAM and NLM were not significantly different. In the case of frames having one member being much more heavily stressed in comparison to the others, both the SRM and DAM should provide significantly more accurate ultimate strength predictions in comparison to the NLM owing to their consideration of the force redistribution resulting from the development of different extents of plasticity within the members of the frame.

\subsection{Application of the stiffness reduction method to Vogel [35] frame}

In this subsection, the application of the SRM to the six-storey Vogel [35] frame is illustrated, and the comparisons of the internal force and capacity predictions obtained through the SRM with those of DAM, NLM and GMNIA are presented. The geometrical and material properties, section sizes, and loading conditions of the six-storey Vogel [35] frame are shown in Fig. 7. Note that the gravity loading acts as uniformly distributed loading on the beams. To make the Vogel [35] frame compatible with the adopted elastic design, the original frame is slightly modified herein, with larger section sizes chosen for some of the beams in comparison to those originally selected by Vogel [35]. Since the loads shown are the factored values given by [35], only the provided load combination will be investigated in this subsection, similar to Maleck [20]. The elastic critical load amplifier of the frame $\alpha_{c r}$ associated with the sway buckling mode is 8.89, indicating that the frame is susceptible to second-order $P-\Delta$ effects according to Eurocode 3 [1].

As the first step of the SRM, Linear Elastic Analysis (LA) of the frame was performed and the stiffness reduction factors of the members were calculated using the first-order 
member forces from the LA. The stiffness reduction factors of each member are presented in Table 9, where $\tau_{M N}^{*}$ are the stiffness reduction factors applied to the flexural stiffnesses. As can be seen from the table, the middle columns located at the first-storey (i.e. C21) and at the second-storey (i.e. C22) have the lowest stiffness reduction factors, thus undergoing the highest rates of plasticity (i.e. stiffness reduction) according to the proposed SRM. Table 9 also illustrates that the upper bound stiffness reduction factor of the frame is equal to 0.8 (i.e. $\left.\tau_{M N, \text { lim }}=\max (0.56,0.8)=0.8\right)$ according to eq. (8). Thus, with the exception of the column members C21, C31, C22, C32 and C23, whose stiffness reduction factors are smaller than $\tau_{M N, l i m}=0.8$, the flexural stiffnesses of all the members are reduced by $\tau_{M N, l i m}=0.8$ in the application of the SRM. In the implementation of the DAM, the flexural stiffnesses of the column members $\mathrm{C} 21$ and $\mathrm{C} 22$ were reduced by factors of 0.79 and 0.89 respectively, while those of others were reduced by a factor of 0.9. For the case of the NLM, of course, the nominal elastic stiffness of the members was taken into account.

The comparison of the maximum bending moments within the members determined through the SRM, DAM and NLM against those of GMNIA is illustrated in Table 10 for the most heavily loaded leeward columns and windward beams, and the middle columns. Note that the maximum bending moments within the members are normalised by the major axis plastic bending moment resistance $M_{y, p l}$ (i.e. $m=M_{y, E d} / M_{y, p l}$ ) in Table 10 and $m_{G M N I A}$ corresponds to the normalised maximum bending moment within a member determined through GMNIA. In this frame, the column member C21 (i.e. the first-storey middle column) experiences the highest rate of plasticity, thus it attracts less gravity bending moment from the beams. Moreover, since the outer columns C11 and C31 remain relatively elastic, their contribution to the lateral stability of the structure increases and the bending moments caused by the wind loading is distributed to these members at a higher rate. The same comments can also be made for the columns in the storeys 2 and 3, where the middle columns experience a higher rate of plasticity in comparison to the outer columns. Since the described different rates of plasticity undergone by the members are taken into account, both the SRM and DAM provide accurate predictions of the internal forces in the members, as can be seen from Table 10. Although the SRM overestimates the internal bending moments within the most heavily stressed member $\mathrm{C} 21$, it provides a more accurate prediction in comparison to the DAM. For the case of the leeward outer column within the first storey (i.e. the C31 member), the SRM considers its relative elasticity and provides a slightly more accurate result than the DAM which leads to the higher underestimation of the bending moments withstood by this member. Since all the members are assumed to be nominally elastic, the NLM does not consider the influence of the development of different rates of plasticity within the members on the internal force distribution and provides less accurate results in comparison to the SRM and the DAM. Particularly, for the C21 member undergoing the largest extent of plasticity, the NLM leads to quite conservative results, overestimating the maximum bending moment resisted by this member by $38 \%$ in comparison to GMNIA.

The design checks of the Vogel [35] frame are carried out through the SRM, DAM and NLM in Table 11 considering its most heavily stressed C21 member. While only the crosssection checks are performed in the case of the SRM, the column buckling curves and beamcolumn design equations of AISC 360-10 [2] and Eurocode 3 [1] are employed in the design 
according to the DAM and NLM. Note that in Table $11, N_{R d}$ is the available axial strength of the member and $1 / \alpha_{u l t, k}$ corresponds to its utilisation rate. In the case of the SRM, $N_{R d}$ is equal to the axial yield load, i.e. $N_{R d}=N_{p l}$, while in the case of the DAM and NLM, it is equal to the axial yield load multiplied by a buckling reduction factor $\chi$ i.e. $N_{R d}=\chi N_{p l}$. As can be seen from Table 11, the member strength of the C21 is violated according to all the SRM, DAM and NLM. Since the DAM and NLM overpredict the bending moments influencing the $\mathrm{C} 21$ member and also use column buckling curves and beam-column equations, the utilisation rate of the $\mathrm{C} 21$ member is significantly higher according to these methods in comparison to that calculated using the SRM.

The ultimate load factors of the Vogel [35] frame $\alpha_{\text {ult }}$ determined through the different design methods, for which the utilisation rate of the critical member $\mathrm{C} 21$ becomes equal to 1.0 (i.e. $1 / \alpha_{u l t, k}=1.0$ ), are compared against that obtained from GMNIA in Table 12 . The $\alpha_{u l t}$ values were determined through iteration in the SRM, DAM and NLM methods as they are based on Geometrically Nonlinear Analysis, and the stiffness reduction factors were recalculated in the case of the SRM and DAM considering the first-order member forces for the load factor $\alpha_{\text {ult }}$. As can be seen from Table 12, the SRM leads to a considerably more accurate strength prediction in comparison to the DAM and NLM, which significantly underpredict the strength of the frame.

\section{Conclusions}

In this paper, a stiffness reduction method (SRM) for the in-plane design of steel frames is presented. The proposed method is implemented by reducing the flexural stiffnesses $(E I)$ of the members of a steel frame through the developed stiffness reduction functions on the basis of the first-order forces they withstand, performing Geometrically Nonlinear Analysis and making cross-section strength checks. Finite element models of the steel members and frames were developed and validated against experimental results from the literature. Geometrically and Materially Nonlinear Analyses with Imperfections (GMNIA) of these finite element models were then employed to verify to proposed SRM in all the considered cases. The proposed approach was initially verified for 2997 simply-supported beam-columns with 42 different loading conditions corresponding to different bending moment shapes observed in steel structures. It was shown that owing to the consideration of the influence of bending moment shape on the development of plasticity within its stiffness reduction reduction functions, the SRM generally leads to considerably more accurate ultimate strength predictions than those of the direct analysis method of AISC 360-10 [2]. In the verification of the proposed SRM for the design of steel frames, the focus was initially placed on sway inhibited and sway uninhibited frames with low redundancy. For the case of the sway inhibited frames with low redundancy, it was observed that the proposed SRM underpredicts bending moments transferred from the beams to the columns. To prevent this underprediction, an upper bound stiffness reduction factor approach was used where the rate of stiffness reduction applied to all the members of a frame, including the beams, must be less than or equal to an upper bound stiffness reduction factor of the frame. The SRM was verified for 817 non-redundant Kanchanalai [28] sway uninhibited frames, some of which also had leaning 
columns generating destabilising effects on the columns providing the lateral stability. The SRM was also validated for frames with high redundancy. The accuracy and safety of the proposed SRM with respect to both internal force and ultimate strength predictions were verified for 16 Ziemian [32] frames with symmetric and unsymmetric geometry and different base support conditions, material properties and loading conditions. The application and accuracy of the SRM was also illustrated for the six-storey Vogel [35] frame. It was observed that the proposed SRM leads to accurate internal force and ultimate strength predictions for the Vogel frame [35] owing to its ability to account for the influence of the development of different rates of plasticity within the members on the internal force distribution of steel frames unlike the notional load method of Eurocode 3 [1].

The proposed SRM, which can be readily applied through conventional structural analysis software, precludes the need of using member design equations, and only requires crosssection strength checks. This feature of the proposed SRM also makes it readily extendible to frames involving members with irregular geometry such as tapered or stepped members or curved members unlike the direct analysis method of AISC 360-10 [2]. The SRM also enables account to be taken of the changes of forces and moments within structural frames depending on the experienced level of stiffness reduction, providing a better insight into the response of the structure in comparison to the conventional steel design where these changes are generally ignored. The SRM proposed in this paper can be directly applied to steel frames sufficiently restrained against out-of-plane instability effects. It is worth noting that in this paper, only frames with members under major axis bending are considered. Future research will be directed towards the application of the SRM to frames with members under minor axis bending [36], a preliminary study into which has been performed by Kucukler [21], the out-of-plane assessment of steel frames by following the proposals of Kucukler et al. $[14,15]$ and the application of the SRM to steel frames with irregular geometries and members.

\section{References}

[1] EN 1993-1-1, Eurocode 3 Design of steel structures-Part 1-1: General rules and rules for buildings. European Committee for Standardization (CEN), Brussels; 2005.

[2] AISC 360-10, Specifications for structural steel buildings. American Institute of Steel Construction (AISC), Chicago; 2010.

[3] Standards Australia, AS 4100 steel structures. Australian Building Codes Board, Sydney; 1998.

[4] Liew, J.Y.R., White, D.W., Chen, W.F.. Second-order refined plastic-hinge analysis for frame design. Part I. Journal of Structural Engineering, ASCE 1993;119(11):3196-3216.

[5] Liew, J.Y.R., White, D.W., Chen, W.F.. Second-order refined plastic-hinge analysis for frame design. Part II. Journal of Structural Engineering, ASCE 1993;119(11):3217-3236.

[6] Ziemian, R.D., McGuire, W.. Modified tangent modulus approach, a contribution to plastic hinge analysis. Journal of Structural Engineering, ASCE 2002;128(10):1301-1307.

[7] Zubydan, A.H.. A simplified model for inelastic second order analysis of planar frames. Engineering Structures 2010;32(10):3258-3268.

[8] Zubydan, A.H.. Inelastic second order analysis of steel frame elements flexed about minor axis. Engineering Structures 2011;33(4):1240-1250.

[9] Kim, S.E., Chen, W.F.. Practical advanced analysis for braced steel frame design. Journal of Structural Engineering, ASCE 1996;122(11):1266-1274. 
[10] Kim, S.E., Chen, W.F.. Practical advanced analysis for unbraced steel frame design. Journal of Structural Engineering, ASCE 1996;122(11):1259-1265.

[11] Surovek-Maleck, A.E., White, D.W.. Alternative approaches for elastic analysis and design of steel frames. I: Overview. Journal of Structural Engineering, ASCE 2004;130(8):1186-1196.

[12] Surovek-Maleck, A.E., White, D.W.. Alternative approaches for elastic analysis and design of steel frames. II: Verification studies. Journal of Structural Engineering, ASCE 2004;130(8):1197-1205.

[13] Kucukler, M., Gardner, L., Macorini, L.. A stiffness reduction method for the in-plane design of structural steel elements. Engineering Structures 2014;73:72-84.

[14] Kucukler, M., Gardner, L., Macorini, L.. Lateral-torsional buckling assessment of steel beams through a stiffness reduction method. Journal of Constructional Steel Research 2015;109:87-100.

[15] Kucukler, M., Gardner, L., Macorini, L.. Flexural-torsional buckling assessment of steel beamcolumns through a stiffness reduction method. Engineering Structures 2015;101:662-676.

[16] Abaqus v.6.10 Reference Manual. Simulia, Dassault Systemes; 2010.

[17] ECCS, Ultimate limit state calculation of sway frames with rigid joints. Tech. Rep.; No. 33, Technical Committee 8 (TC 8) of European Convention for Constructional Steelwork (ECCS); 1984.

[18] AISC Code of Standard Practice for Steel Buildings and Bridges. American Institute of Steel Construction; 2010.

[19] Ziemian, R.D., McGuire, W., Deierlein, G.G.. Inelastic limit states design. Part I: Planar frame studies. Journal of Structural Engineering, ASCE 1992;118(9):2532-2549.

[20] Maleck, A.E.. Second-order inelastic and modified elastic analysis and design evaluation of planar steel frames. Ph.D. thesis; Georgia Institute of Technology; 2001.

[21] Kucukler, M.. Stiffness reduction approach for structural steel design. Ph.D. thesis; Imperial College London; 2015.

[22] Austin, W.J.. Strength and design of metal beam-columns. Journal of the Structural Division 1961;87(4):1-32.

[23] Kirby, P.A., Nethercot, D.A.. Design for structural stability. John Wiley \& Sons, London; 1979.

[24] Duan, L., Chen, W.F.. A yield surface equation for doubly symmetrical sections. Engineering Structures 1990;12(2):114-119.

[25] EN-1090-2: Execution of steel structures and aluminium structures-Part 2: Technical requirements for steel structures. European Committee for Standardization (CEN), Brussels; 2008.

[26] Matlab version 8.5.0 (R2015a). Mathworks Inc.; 2015.

[27] Chen, W., Toma, S.. Advanced analysis of steel frames: theory, software, and applications. CRC press; 1994.

[28] Kanchanalai, T.. The design and behavior of beam-columns in unbraced steel frames. AISI Project No. 189. Tech. Rep.; The University of Texas at Austin; 1977.

[29] ASCE, Effective length and notional load approaches for assessing frame stability: Implications for American steel design. ASCE technical committee on LRFD design, Committee report, New York; 1997.

[30] Boissonnade, N., Greiner, R., Jaspart, J.P., Lindner, J.. Rules for Member Stability in EN 1993-1-1: Background documentation and design guidelines, No 119. ECCS European Convention for Constructional Steelwork; 2006.

[31] Ziemian, R.D.. Mastan2 v3.3. Interactive structural analysis program. http://www.mastan2.com/index (Accessed: 2016-11-03); 2016.

[32] Ziemian, R.D.. Advanced methods of inelastic analysis in the limit states design of steel structures. Ph.D. thesis; Cornell University; 1990.

[33] LRFD, Load and resistance factor design specification for structural steel buildings. American Institute of Steel Construction (AISC), Chicago; 1986.

[34] EN-1990, Eurocode - Basis of structural design. European Committee for Standardization (CEN), Brussels; 2002.

[35] Vogel, U.. Calibrating frames. Stahlbau 1985;54:293-301.

[36] Ziemian, R.D., Miller, A.R.. Inelastic analysis and design: frames with members in minor-axis 
bending. Journal of Structural Engineering, ASCE 1997;123(2):151-156. 


\section{Tables captions}

Table 1 : Imperfection factors $\alpha$ for the stiffness reduction function for I-sections under axial loading and buckling about the major axis

Table 2: Parameters for the stiffness reduction function for I-sections in major axis bending $\tau_{M}[7,8]$

Table 3 : Considered load cases for the assessment of the proposed stiffness reduction method - Negative values of $\mu$ and $\xi$ indicate that the applied moment is in the opposite direction to that shown

Table 4 : Comparison of the accuracy of the proposed method (SRM) against GMNIA, the direct analysis method (DAM) of AISC 360-10 [2] and the beam-column design methods provided in Annex A (EC3-A) and Annex B (EC3-B) of Eurocode 3 [1] for beam-columns under various loading conditions

Table 5 : Considered section types for the studied non-sway frames

Table 6 : Comparison of the accuracy of different design methods for the prediction of internal forces at the top of the columns and strengths of pin-based sway-inhibited frames

Table 7 : Comparison of the accuracy of different design methods against GMNIA for Kanchalanai [28] frames

Table 8 : Comparison of the strengths and internal forces determined through the proposed stiffness reduction method (SRM), the direct analysis method (DAM) and the notional load method (NLM) against those obtained through GMNIA

Table 9 : Stiffness reduction factors calculated for members of the Vogel [35] frame

Table 10 : Maximum normalised bending moments within members determined using the different design methods for the Vogel [35] frame - $m=M_{y, E d} / M_{y, p l}$

Table 11 : Design checks of the members of the Vogel [35] frame according to different design methods

Table 12 : Ultimate load factors of the Vogel [35] frame determined through different design methods 
Table 1: Imperfection factors $\alpha$ for the stiffness reduction function for I-sections under axial loading and buckling about the major axis

\begin{tabular}{cc}
\hline Aspect ratio of the cross-section & $\alpha$ \\
$h / b \leq 1.2$ & 0.26 \\
$h / b>1.2$ & 0.21 \\
\hline
\end{tabular}

Table 2: Parameters for the stiffness reduction function for I-sections in major axis bending $\tau_{M}[7,8]$

\begin{tabular}{cccccc}
\hline & $\tau_{M l}$ & $\phi_{y}$ & $\xi_{m}$ & $\beta_{m}$ & $\delta_{m}$ \\
\cline { 2 - 5 }$h / b \leq 1.2$ & 0.04 & $0.5 \frac{W e l, y}{W p l, y}$ & 0.98 & 1.5 & 1.0 \\
$h / b>1.2$ & 0.08 & $0.7 \frac{W e l, y}{W p l, y}$ & 0.95 & 1.5 & 1.0 \\
\hline
\end{tabular}


Table 3: Considered load cases for the assessment of the proposed stiffness reduction method - Negative values of $\mu$ and $\xi$ indicate that the applied moment is in the opposite direction to that shown

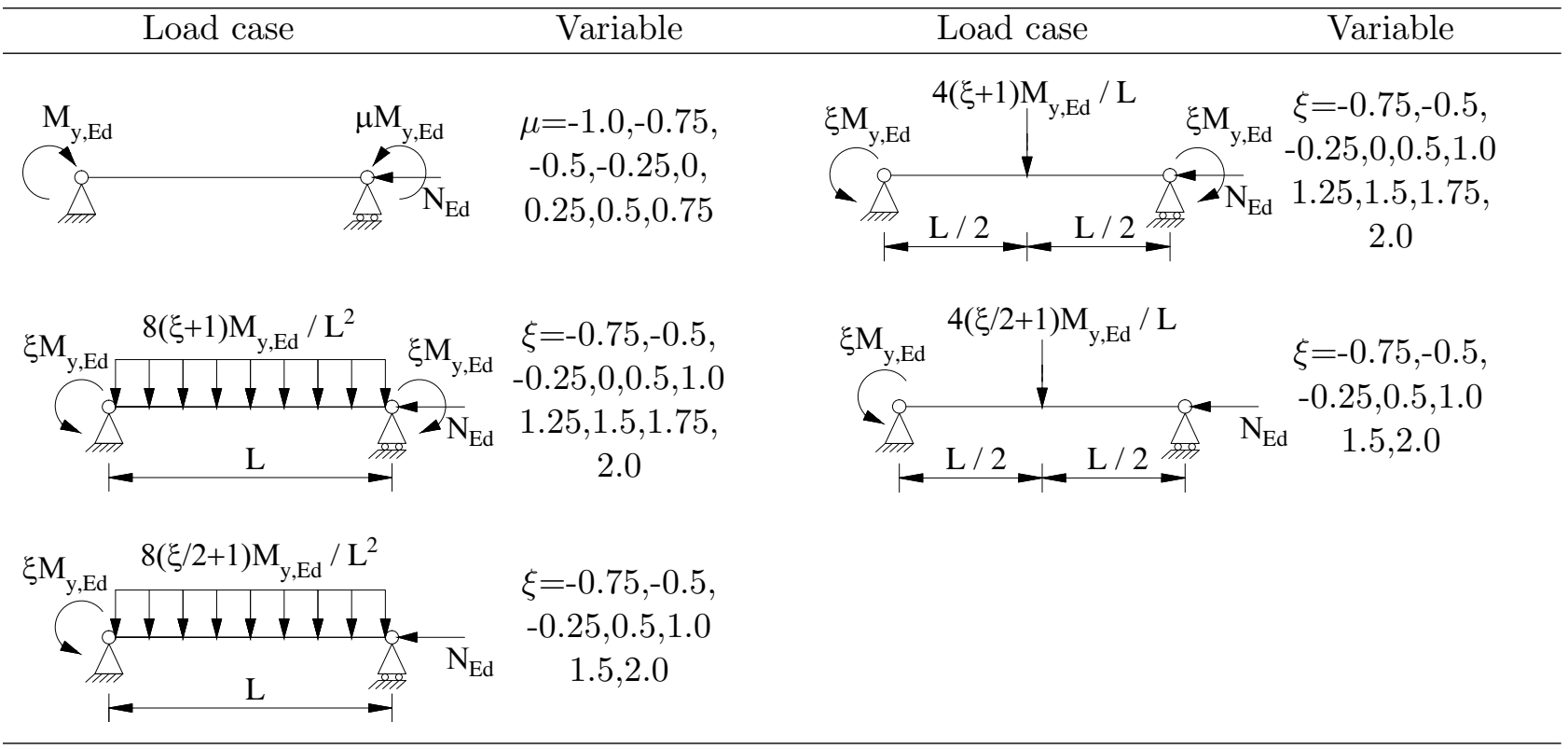


Table 4: Comparison of the accuracy of the proposed method (SRM) against GMNIA, the direct analysis method (DAM) of AISC 360-10 [2] and the beam-column design methods provided in Annex A (EC3-A) and Annex B (EC3-B) of Eurocode 3 [1] for beam-columns under various loading conditions

\begin{tabular}{|c|c|c|c|c|c|c|}
\hline Load case & $\mathrm{N}$ & Method & $\epsilon_{a v}$ & $\epsilon_{\text {cov }}$ & $\epsilon_{\max }$ & $\epsilon_{\min }$ \\
\hline \multirow{4}{*}{$\int_{\text {mा }}^{\mathrm{M}_{\mathrm{y}, \mathrm{Ed}}}$} & \multirow{4}{*}{543} & SRM & 1.00 & 0.017 & 1.06 & 0.97 \\
\hline & & DAM & 0.93 & 0.067 & 1.06 & 0.68 \\
\hline & & EC3-A & 0.96 & 0.029 & 1.01 & 0.89 \\
\hline & & EC3-B & 0.96 & 0.041 & 1.03 & 0.77 \\
\hline \multirow{4}{*}{ 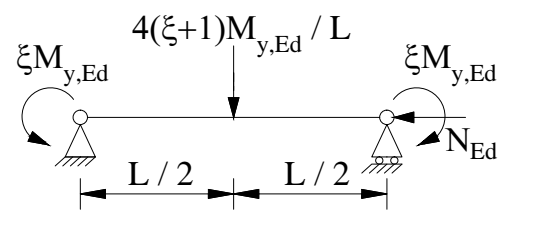 } & \multirow{4}{*}{694} & SRM & 1.00 & 0.021 & 1.06 & 0.91 \\
\hline & & DAM & 0.93 & 0.069 & 1.06 & 0.66 \\
\hline & & EC3-A & 0.95 & 0.044 & 1.01 & 0.75 \\
\hline & & EC3-B & 0.94 & 0.060 & 1.01 & 0.71 \\
\hline \multirow{4}{*}{$\xi \mathrm{M}_{\mathrm{y}, \mathrm{Ed}}^{8(\xi+1) \mathrm{M}_{\mathrm{y}, \mathrm{Ed}} / \mathrm{L}^{2}} \mathrm{M}_{\mathrm{y}, \mathrm{Ed}}$} & \multirow{4}{*}{689} & SRM & 1.00 & 0.017 & 1.05 & 0.95 \\
\hline & & DAM & 0.95 & 0.053 & 1.06 & 0.79 \\
\hline & & EC3-A & 0.96 & 0.042 & 1.01 & 0.76 \\
\hline & & EC3-B & 0.96 & 0.031 & 1.01 & 0.84 \\
\hline \multirow{4}{*}{ 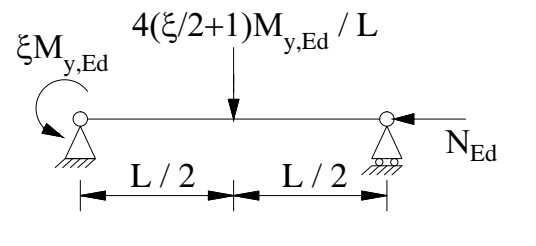 } & \multirow{4}{*}{519} & SRM & 1.00 & 0.016 & 1.05 & 0.96 \\
\hline & & DAM & 0.93 & 0.056 & 1.06 & 0.75 \\
\hline & & EC3-A & 0.96 & 0.041 & 1.01 & 0.77 \\
\hline & & EC3-B & 0.95 & 0.035 & 1.04 & 0.85 \\
\hline \multirow{4}{*}{$\xi \mathrm{M}_{\mathrm{y}, \mathrm{Ed}} 8(\xi / 2+1) \mathrm{M}_{\mathrm{y}, \mathrm{Ed}} / \mathrm{L}^{2}$} & \multirow{4}{*}{552} & SRM & 1.00 & 0.018 & 1.05 & 0.95 \\
\hline & & DAM & 0.95 & 0.050 & 1.06 & 0.83 \\
\hline & & EC3-A & 0.97 & 0.035 & 1.01 & 0.77 \\
\hline & & EC3-B & 0.97 & 0.022 & 1.01 & 0.89 \\
\hline
\end{tabular}


Table 5: Considered section types for the studied non-sway frames

\begin{tabular}{cccc}
\hline Column section & Beam section & $G_{R}$ & $M_{y, p l, c} / M_{y, p l, b}$ \\
& HEB900 & 0.35 & 0.25 \\
HEB400 & HEB600 & 1.01 & 0.50 \\
& HEB400 & 3.00 & 1.00 \\
\hline \multirow{3}{*}{ HEB200 } & IPE500 & 0.36 & 0.29 \\
& IPE360 & 1.07 & 0.64 \\
& IPE270 & 3.00 & 1.35 \\
\hline
\end{tabular}

Table 6: Comparison of the accuracy of different design methods for the prediction of internal forces at the top of the columns and strengths of pin-based sway-inhibited frames

\begin{tabular}{cccccccc}
\hline$G_{R}$ & $\mathrm{~N}$ & Method & $\epsilon_{\max }$ & $\zeta_{\text {av }}$ & $\zeta_{\text {cov }}$ & $\zeta_{\max }$ & $\zeta_{\min }$ \\
& & SRM & 1.06 & 0.92 & 0.045 & 1.00 & 0.83 \\
$0.35-0.36$ & 54 & SRM-Limit & 1.02 & 0.99 & 0.025 & 1.04 & 0.93 \\
& & DAM & 0.99 & 1.01 & 0.034 & 1.11 & 0.95 \\
\hline \multirow{3}{*}{$1.01-1.07$} & \multirow{2}{*}{54} & SRM & 1.05 & 0.97 & 0.024 & 1.00 & 0.91 \\
& & SRM-Limit & 1.02 & 1.00 & 0.010 & 1.02 & 0.98 \\
& & DAM & 0.96 & 1.01 & 0.013 & 1.05 & 0.99 \\
\hline \multirow{3}{*}{3.00} & \multirow{2}{*}{54} & SRM & 1.05 & 0.99 & 0.017 & 1.03 & 0.95 \\
& & SRM-Limit & 1.03 & 1.01 & 0.010 & 1.04 & 0.99 \\
& & DAM & 0.94 & 1.01 & 0.011 & 1.04 & 0.99 \\
\hline
\end{tabular}


Table 7: Comparison of the accuracy of different design methods against GMNIA for Kanchalanai [28] frames

\begin{tabular}{|c|c|c|c|c|c|c|c|c|c|c|c|c|c|c|c|c|c|c|c|}
\hline \multirow[b]{2}{*}{ Frame } & \multirow[b]{2}{*}{$G_{R}$} & \multirow[b]{2}{*}{$\gamma_{L}$} & \multirow[b]{2}{*}{$\mathrm{N}$} & \multicolumn{4}{|c|}{ SRM } & \multicolumn{4}{|c|}{ DAM } & \multicolumn{4}{|c|}{ NLM EC3-A } & \multicolumn{4}{|c|}{ NLM EC3-B } \\
\hline & & & & $\epsilon_{a v}$ & $\epsilon_{c o v}$ & $\epsilon_{\max }$ & $\epsilon_{\min }$ & $\epsilon_{a v}$ & $\epsilon_{c o v}$ & $\epsilon_{\max }$ & $\epsilon_{\min }$ & $\epsilon_{a v}$ & $\epsilon_{c o v}$ & $\epsilon_{\max }$ & $\epsilon_{\min }$ & $\epsilon_{a v}$ & $\epsilon_{c o v}$ & $\epsilon_{\max }$ & $\epsilon_{\min }$ \\
\hline \multirow{3}{*}{$\mathrm{FP}$} & 0 & & 45 & 0.98 & 0.028 & 1.02 & 0.91 & 0.99 & 0.028 & 1.07 & 0.93 & 0.95 & 0.028 & 0.99 & 0.87 & 0.94 & 0.031 & 1.00 & 0.86 \\
\hline & 1 & & 42 & 0.97 & 0.034 & 1.02 & 0.87 & 0.99 & 0.024 & 1.03 & 0.93 & 0.95 & 0.029 & 0.99 & 0.88 & 0.94 & 0.028 & 0.97 & 0.86 \\
\hline & 3 & & 38 & 0.96 & 0.039 & 1.01 & 0.88 & 0.98 & 0.024 & 1.00 & 0.91 & 0.94 & 0.029 & 0.98 & 0.88 & 0.94 & 0.028 & 0.97 & 0.87 \\
\hline \multirow{3}{*}{$\mathrm{FR}$} & 0 & & 54 & 0.99 & 0.030 & 1.03 & 0.91 & 0.94 & 0.032 & 0.98 & 0.85 & 0.94 & 0.028 & 0.98 & 0.87 & 0.92 & 0.025 & 0.97 & 0.85 \\
\hline & 1 & & 51 & 0.97 & 0.036 & 1.01 & 0.87 & 0.94 & 0.022 & 0.98 & 0.90 & 0.92 & 0.031 & 0.96 & 0.86 & 0.91 & 0.020 & 0.93 & 0.85 \\
\hline & 3 & & 42 & 0.92 & 0.059 & 0.99 & 0.80 & 0.93 & 0.036 & 0.97 & 0.84 & 0.90 & 0.030 & 0.94 & 0.85 & 0.90 & 0.022 & 0.92 & 0.85 \\
\hline \multirow{6}{*}{ LFP } & 0 & 1 & 36 & 0.98 & 0.023 & 1.02 & 0.93 & 1.01 & 0.026 & 1.07 & 0.95 & 0.94 & 0.023 & 0.97 & 0.87 & 0.93 & 0.017 & 0.96 & 0.89 \\
\hline & 0 & 2 & 50 & 0.98 & 0.019 & 1.01 & 0.94 & 1.00 & 0.022 & 1.04 & 0.95 & 0.93 & 0.025 & 0.97 & 0.85 & 0.92 & 0.022 & 0.97 & 0.87 \\
\hline & 0 & 3 & 54 & 0.98 & 0.017 & 1.01 & 0.94 & 1.00 & 0.020 & 1.04 & 0.95 & 0.93 & 0.033 & 0.98 & 0.85 & 0.92 & 0.033 & 0.98 & 0.83 \\
\hline & 2 & 1 & 29 & 0.97 & 0.025 & 1.00 & 0.91 & 0.99 & 0.022 & 1.02 & 0.94 & 0.94 & 0.020 & 0.97 & 0.90 & 0.93 & 0.022 & 0.98 & 0.90 \\
\hline & 2 & 2 & 40 & 0.97 & 0.020 & 1.00 & 0.90 & 0.99 & 0.026 & 1.02 & 0.93 & 0.94 & 0.026 & 0.98 & 0.86 & 0.93 & 0.030 & 0.99 & 0.86 \\
\hline & 2 & 3 & 42 & 0.96 & 0.027 & 1.01 & 0.90 & 0.98 & 0.023 & 1.02 & 0.94 & 0.94 & 0.031 & 0.99 & 0.84 & 0.94 & 0.034 & 1.00 & 0.84 \\
\hline \multirow{6}{*}{ LFR } & 0 & 1 & 42 & 0.98 & 0.030 & 1.02 & 0.91 & 0.96 & 0.021 & 0.99 & 0.92 & 0.90 & 0.034 & 0.93 & 0.82 & 0.91 & 0.021 & 0.93 & 0.85 \\
\hline & 0 & 2 & 63 & 0.98 & 0.024 & 1.02 & 0.92 & 0.98 & 0.016 & 1.00 & 0.94 & 0.89 & 0.038 & 0.95 & 0.79 & 0.90 & 0.026 & 0.95 & 0.82 \\
\hline & 0 & 3 & 67 & 0.98 & 0.020 & 1.01 & 0.92 & 0.98 & 0.016 & 1.01 & 0.94 & 0.90 & 0.045 & 0.96 & 0.78 & 0.90 & 0.034 & 0.96 & 0.82 \\
\hline & 2 & 1 & 32 & 0.93 & 0.049 & 0.98 & 0.83 & 0.95 & 0.038 & 0.99 & 0.84 & 0.88 & 0.031 & 0.93 & 0.84 & 0.90 & 0.022 & 0.94 & 0.85 \\
\hline & 2 & 2 & 44 & 0.93 & 0.043 & 0.99 & 0.85 & 0.95 & 0.040 & 0.99 & 0.84 & 0.89 & 0.035 & 0.96 & 0.83 & 0.91 & 0.027 & 0.96 & 0.85 \\
\hline & 2 & 3 & 46 & 0.94 & 0.041 & 0.99 & 0.86 & 0.95 & 0.041 & 0.99 & 0.84 & 0.90 & 0.038 & 0.97 & 0.84 & 0.91 & 0.029 & 0.97 & 0.86 \\
\hline
\end{tabular}


Table 8: Comparison of the strengths and internal forces determined through the proposed stiffness reduction method (SRM), the direct analysis method (DAM) and the notional load method (NLM) against those obtained through GMNIA

\begin{tabular}{|c|c|c|c|c|c|c|c|c|c|c|c|c|c|c|c|c|c|}
\hline \multirow[b]{2}{*}{ Frame } & \multirow[b]{2}{*}{$\alpha_{c r}$} & \multirow{2}{*}{$\frac{\text { GMNIA }}{\alpha_{u l t}}$} & \multicolumn{5}{|c|}{ SRM } & \multicolumn{5}{|c|}{ DAM } & \multicolumn{5}{|c|}{ NLM } \\
\hline & & & $\alpha_{u l t}$ & $\zeta_{a v}$ & $\zeta_{c o v}$ & $\zeta_{\max }$ & $\zeta_{\text {min }}$ & $\alpha_{u l t}$ & $\zeta_{a v}$ & $\zeta_{c o v}$ & $\zeta_{\max }$ & $\zeta_{\min }$ & $\alpha_{u l t}$ & $\zeta_{a v}$ & $\zeta_{c o v}$ & $\zeta_{\max }$ & $\zeta_{\min }$ \\
\hline UP36L & 6.85 & 1.28 & 0.93 & 1.02 & 0.028 & 1.08 & 0.98 & 0.93 & 1.01 & 0.029 & 1.08 & 0.98 & 0.92 & 1.03 & 0.029 & 1.09 & 0.99 \\
\hline UP36H & 3.85 & 1.14 & 0.98 & 1.01 & 0.022 & 1.04 & 0.96 & 0.98 & 1.00 & 0.015 & 1.02 & 0.98 & 0.99 & 1.03 & 0.019 & 1.06 & 1.01 \\
\hline UF36L & 13.68 & 1.29 & 0.97 & 1.01 & 0.019 & 1.04 & 0.98 & 0.97 & 1.01 & 0.017 & 1.04 & 0.98 & 0.97 & 1.02 & 0.016 & 1.04 & 1.00 \\
\hline UF36H & 14.04 & 1.25 & 1.00 & 1.01 & 0.018 & 1.03 & 0.97 & 1.00 & 1.01 & 0.015 & 1.02 & 0.98 & 1.01 & 1.02 & 0.018 & 1.05 & 0.99 \\
\hline UP50L & 7.22 & 1.24 & 1.00 & 1.01 & 0.019 & 1.05 & 0.98 & 0.99 & 1.01 & 0.012 & 1.02 & 0.98 & 1.01 & 1.02 & 0.021 & 1.07 & 0.99 \\
\hline UP50H & 2.97 & 1.14 & 0.98 & 1.02 & 0.021 & 1.04 & 0.97 & 0.98 & 1.00 & 0.022 & 1.03 & 0.97 & 0.97 & 1.03 & 0.018 & 1.06 & 1.00 \\
\hline UF50L & 11.14 & 1.29 & 0.95 & 1.01 & 0.023 & 1.05 & 0.97 & 0.95 & 1.01 & 0.022 & 1.06 & 0.97 & 0.95 & 1.02 & 0.021 & 1.06 & 0.98 \\
\hline $\mathrm{UF} 50 \mathrm{H}$ & 9.95 & 1.22 & 0.99 & 1.01 & 0.023 & 1.04 & 0.98 & 0.98 & 1.01 & 0.019 & 1.03 & 0.97 & 0.98 & 1.03 & 0.025 & 1.09 & 1.00 \\
\hline SP36L & 7.09 & 1.27 & 0.90 & 1.01 & 0.066 & 1.11 & 0.91 & 0.90 & 1.01 & 0.065 & 1.11 & 0.91 & 0.89 & 1.04 & 0.067 & 1.13 & 0.95 \\
\hline SP36H & 2.39 & 1.05 & 1.00 & 1.03 & 0.029 & 1.08 & 1.00 & 1.02 & 1.00 & 0.011 & 1.01 & 0.99 & 1.00 & 1.03 & 0.025 & 1.06 & 1.00 \\
\hline SF36L & 9.44 & 1.24 & 1.00 & 1.01 & 0.016 & 1.03 & 0.99 & 1.00 & 1.01 & 0.013 & 1.03 & 0.99 & 1.00 & 1.02 & 0.013 & 1.03 & 1.00 \\
\hline SF36H & 7.92 & 1.19 & 1.00 & 1.01 & 0.013 & 1.03 & 1.00 & 1.00 & 1.01 & 0.013 & 1.03 & 1.00 & 1.01 & 1.02 & 0.017 & 1.05 & 1.00 \\
\hline SP50L & 7.38 & 1.68 & 1.15 & 1.01 & 0.003 & 1.01 & 1.00 & 1.14 & 1.01 & 0.002 & 1.01 & 1.00 & 1.16 & 1.03 & 0.021 & 1.07 & 1.01 \\
\hline $\mathrm{SP} 50 \mathrm{H}$ & 1.96 & 1.08 & 0.97 & 1.04 & 0.040 & 1.10 & 1.00 & 0.98 & 1.01 & 0.009 & 1.03 & 1.00 & 0.98 & 1.04 & 0.034 & 1.09 & 1.01 \\
\hline SF50L & 8.08 & 1.39 & 0.98 & 1.01 & 0.017 & 1.04 & 1.00 & 0.98 & 1.01 & 0.016 & 1.03 & 0.99 & 0.98 & 1.02 & 0.011 & 1.04 & 1.01 \\
\hline $\mathrm{SF} 50 \mathrm{H}$ & 5.81 & 1.20 & 1.00 & 1.01 & 0.012 & 1.02 & 1.00 & 1.00 & 1.01 & 0.013 & 1.03 & 1.01 & 1.02 & 1.02 & 0.017 & 1.05 & 1.00 \\
\hline
\end{tabular}


Table 9: Stiffness reduction factors calculated for members of the Vogel [35] frame

\begin{tabular}{ccccccccccc}
\hline Member & $\tau_{N}$ & $C_{m}$ & $\tau_{M}$ & $\tau_{M N}$ & $\tau_{M N}^{*}$ & Member & $C_{m}$ & $\tau_{M}$ & $\tau_{M N}$ & $\tau_{M N}^{*}$ \\
$\mathrm{C} 11$ & 0.83 & 0.31 & 1.00 & 0.80 & 0.80 & $\mathrm{~B} 11$ & 0.21 & 1.00 & 0.99 & 0.80 \\
$\mathrm{C} 21$ & 0.60 & 0.22 & 1.00 & 0.56 & 0.56 & $\mathrm{~B} 21$ & 0.23 & 1.00 & 0.99 & 0.80 \\
$\mathrm{C} 31$ & 0.78 & 0.20 & 1.00 & 0.75 & 0.75 & & & & & \\
\hline $\mathrm{C} 12$ & 0.85 & 0.22 & 1.00 & 0.84 & 0.80 & $\mathrm{~B} 12$ & 0.21 & 1.00 & 0.98 & 0.80 \\
$\mathrm{C} 22$ & 0.70 & 0.20 & 1.00 & 0.67 & 0.67 & $\mathrm{~B} 22$ & 0.20 & 1.00 & 0.99 & 0.80 \\
$\mathrm{C} 32$ & 0.82 & 0.20 & 1.00 & 0.78 & 0.78 & & & & & \\
\hline $\mathrm{C} 13$ & 0.87 & 0.21 & 1.00 & 0.86 & 0.80 & $\mathrm{~B} 13$ & 0.20 & 1.00 & 0.98 & 0.80 \\
$\mathrm{C} 23$ & 0.75 & 0.20 & 1.00 & 0.72 & 0.72 & $\mathrm{~B} 23$ & 0.20 & 1.00 & 0.99 & 0.80 \\
$\mathrm{C} 33$ & 0.85 & 0.20 & 1.00 & 0.82 & 0.80 & & & & & \\
\hline $\mathrm{C} 14$ & 0.89 & 0.21 & 1.00 & 0.88 & 0.80 & $\mathrm{~B} 14$ & 0.22 & 1.00 & 0.96 & 0.80 \\
$\mathrm{C} 24$ & 0.81 & 0.20 & 1.00 & 0.80 & 0.80 & $\mathrm{~B} 24$ & 0.33 & 1.00 & 0.97 & 0.80 \\
$\mathrm{C} 34$ & 0.88 & 0.21 & 1.00 & 0.86 & 0.80 & & & & & \\
\hline $\mathrm{C} 15$ & 0.89 & 0.21 & 1.00 & 0.88 & 0.80 & $\mathrm{~B} 15$ & 0.26 & 1.00 & 0.98 & 0.80 \\
$\mathrm{C} 25$ & 0.83 & 0.21 & 1.00 & 0.82 & 0.80 & $\mathrm{~B} 25$ & 0.39 & 1.00 & 0.99 & 0.80 \\
$\mathrm{C} 35$ & 0.88 & 0.21 & 1.00 & 0.86 & 0.80 & & & & & \\
\hline $\mathrm{C} 16$ & 0.93 & 0.21 & 1.00 & 0.92 & 0.80 & $\mathrm{~B} 16$ & 0.29 & 1.00 & 0.96 & 0.80 \\
$\mathrm{C} 26$ & 0.91 & 0.22 & 1.00 & 0.91 & 0.80 & $\mathrm{~B} 26$ & 0.33 & 1.00 & 0.96 & 0.80 \\
$\mathrm{C} 36$ & 0.93 & 0.21 & 1.00 & 0.92 & 0.80 & & & & & \\
\hline$\tau_{M N, m i n}=0.56$, & $\tau_{M N, l i m}=\max (0.56,0.8)=0.80$ & & & & &
\end{tabular}


Table 10: Maximum normalised bending moments within members determined using the different design methods for the Vogel [35] frame - $m=M_{y, E d} / M_{y, p l}$

\begin{tabular}{|c|c|c|c|c|c|c|c|}
\hline \multirow{3}{*}{$\begin{array}{c}\text { Member } \\
\text { C21 }\end{array}$} & \multirow{2}{*}{$\begin{array}{c}\text { GMNIA } \\
m\end{array}$} & \multicolumn{2}{|r|}{ SRM } & \multicolumn{2}{|r|}{$\overline{D A M}$} & \multicolumn{2}{|r|}{ NLM } \\
\hline & & $m$ & $m / m_{G M N I A}$ & $m$ & $m / m_{G M N I A}$ & $m$ & $m / m_{G M N I A}$ \\
\hline & 0.368 & 0.415 & 1.13 & 0.446 & 1.21 & 0.508 & 1.38 \\
\hline C31 & 0.544 & 0.536 & 0.99 & 0.515 & 0.95 & 0.523 & 0.96 \\
\hline B11 & 0.922 & 0.949 & 1.03 & 0.938 & 1.02 & 0.965 & 1.05 \\
\hline $\mathrm{C} 22$ & 0.371 & 0.375 & 1.01 & 0.373 & 1.00 & 0.396 & 1.07 \\
\hline C32 & 0.553 & 0.560 & 1.01 & 0.553 & 1.00 & 0.576 & 1.04 \\
\hline B12 & 0.847 & 0.871 & 1.03 & 0.860 & 1.02 & 0.881 & 1.04 \\
\hline $\mathrm{C} 23$ & 0.308 & 0.312 & 1.01 & 0.314 & 1.02 & 0.336 & 1.09 \\
\hline C33 & 0.518 & 0.519 & 1.00 & 0.515 & 1.00 & 0.531 & 1.03 \\
\hline B13 & 0.925 & 0.956 & 1.03 & 0.941 & 1.02 & 0.961 & 1.04 \\
\hline C24 & 0.233 & 0.238 & 1.02 & 0.234 & 1.00 & 0.251 & 1.08 \\
\hline C34 & 0.558 & 0.551 & 0.99 & 0.558 & 1.00 & 0.571 & 1.02 \\
\hline B14 & 0.880 & 0.910 & 1.03 & 0.892 & 1.01 & 0.907 & 1.03 \\
\hline $\mathrm{C} 25$ & 0.258 & 0.262 & 1.01 & 0.259 & 1.00 & 0.279 & 1.08 \\
\hline C35 & 0.619 & 0.611 & 0.99 & 0.621 & 1.00 & 0.637 & 1.03 \\
\hline B15 & 0.840 & 0.870 & 1.03 & 0.854 & 1.02 & 0.862 & 1.03 \\
\hline $\mathrm{C} 26$ & 0.082 & 0.083 & 1.01 & 0.082 & 1.00 & 0.090 & 1.10 \\
\hline C36 & 0.616 & 0.602 & 0.98 & 0.614 & 1.00 & 0.621 & 1.01 \\
\hline B16 & 0.839 & 0.864 & 1.03 & 0.850 & 1.01 & 0.854 & 1.02 \\
\hline \multicolumn{3}{|c|}{$\left(m / m_{G M N I A}\right)_{a v}$} & 1.02 & & 1.02 & & 1.06 \\
\hline & \multicolumn{2}{|c|}{$\left(m / m_{G M N I A}\right)_{c o v}$} & 0.032 & & 0.051 & & 0.082 \\
\hline & \multicolumn{2}{|c|}{$\left(m / m_{G M N I A}\right)_{\max }$} & 1.13 & & 1.21 & & 1.38 \\
\hline & \multicolumn{2}{|c|}{$\left(m / m_{G M N I A}\right)_{\min }$} & 0.98 & & 0.95 & & 0.96 \\
\hline
\end{tabular}


Table 11: Design checks of the members of the Vogel [35] frame according to different design methods

\begin{tabular}{cccccc}
\hline Member & & SRM & DAM & NLM EC3 Annex A & NLM EC3 Annex B \\
& $N_{E d} / N_{R d}$ & 0.68 & 0.71 & 0.68 & 0.68 \\
C21 & $M_{y, E d} / M_{y, p l}$ & 0.42 & 0.45 & 0.51 & 0.51 \\
& $1 / \alpha_{u l t, k}$ & 1.02 & 1.10 & 1.13 & 1.14 \\
\hline
\end{tabular}

Table 12: Ultimate load factors of the Vogel [35] frame determined through different design methods

\begin{tabular}{cccccc}
\hline & GMNIA & SRM & DAM & NLM EC3 Annex A & NLM EC3 Annex B \\
$\alpha_{\text {ult }}$ & 1.106 & 0.984 & 0.894 & 0.887 & 0.884 \\
\hline
\end{tabular}




\section{Figures captions}

Figure 1: Material stress-strain curves used in finite element models

Figure 2 : Residual stress patterns applied to finite element models $(+\mathrm{ve}=$ tension; - ve $=$ compression)

Figure 3 : Rigid-jointed sway-inhihibited frame

Figure 4 : Non-redundant benchmark frames (overturning effects are negligible $L_{b} \gg L$ )

Figure 5: Comparison of the proposed stiffness reduction method (SRM) against GMNIA, the direct analysis method (DAM) and the notional load method with the Eurocode 3 Annex A (NLM EC3-A) and Annex B (NLM EC3-B) beam-column design methods for the non-redundant Kanchanalai [28] frames

Figure 6 : Geometrical properties and loading conditions of the investigated Ziemian [32] frames

Figure 7 : Geometrical and material properties and loading conditions of the modified Vogel [35] frame 


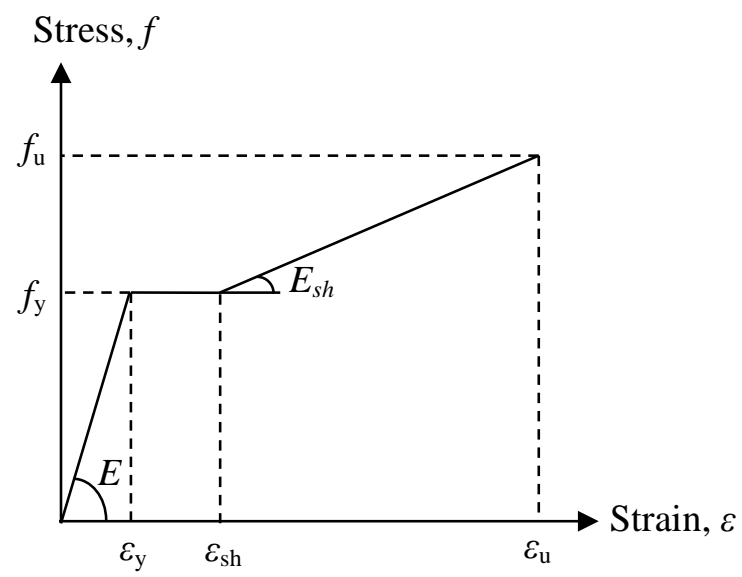

Figure 1: Material stress-strain curves used in finite element models

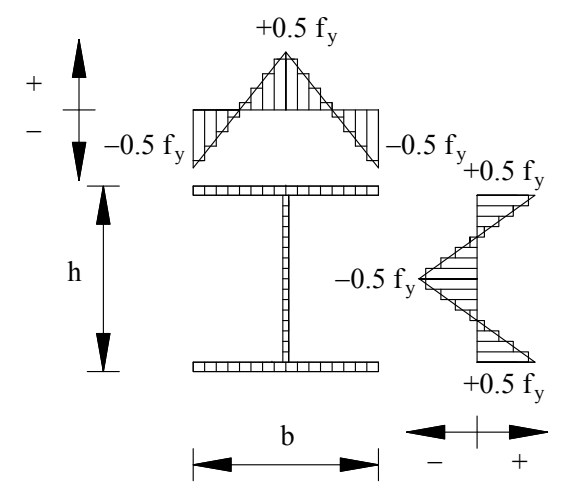

(a) h / b $\leq 1.2$

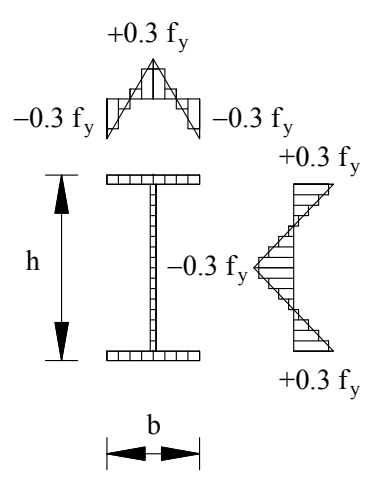

(b) $\mathrm{h} / \mathrm{b}>1.2$

Figure 2: Residual stress patterns applied to finite element models $(+\mathrm{ve}=$ tension; $-\mathrm{ve}=$ compression $)$ 


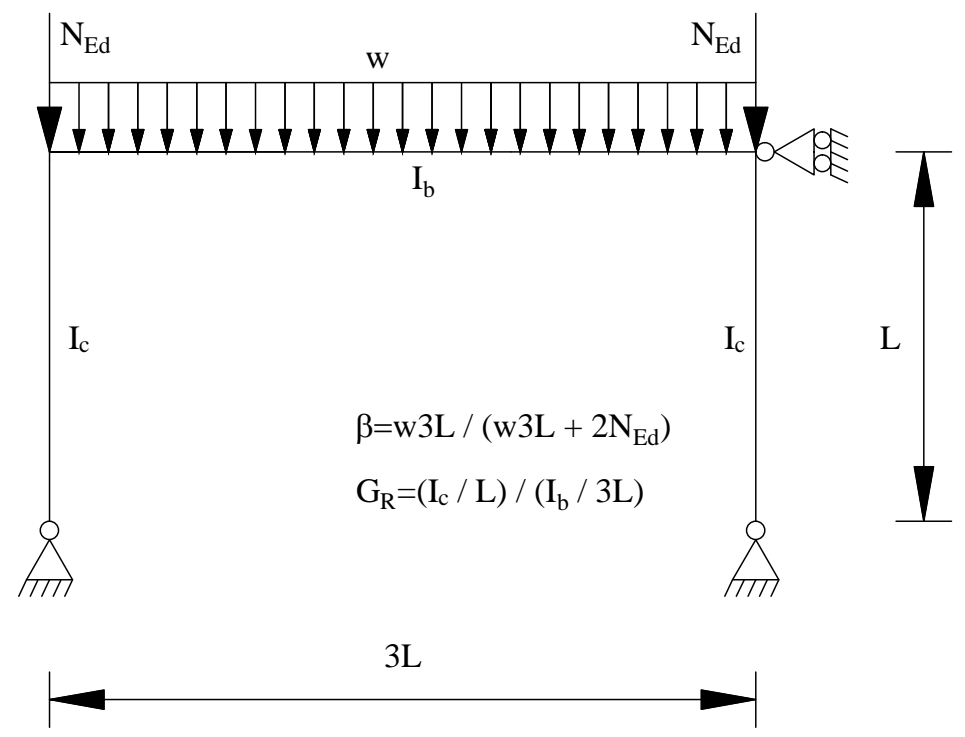

Figure 3: Rigid-jointed sway-inhihibited frame 


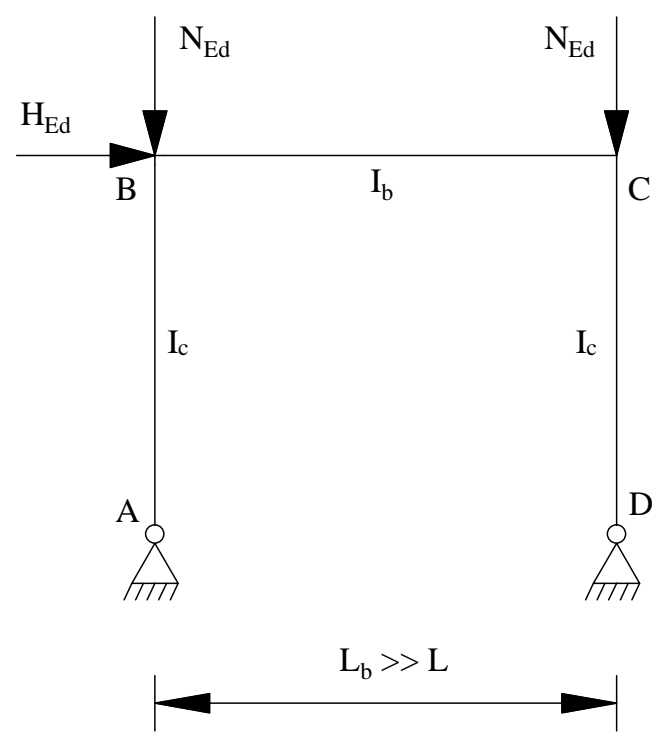

(a) FP
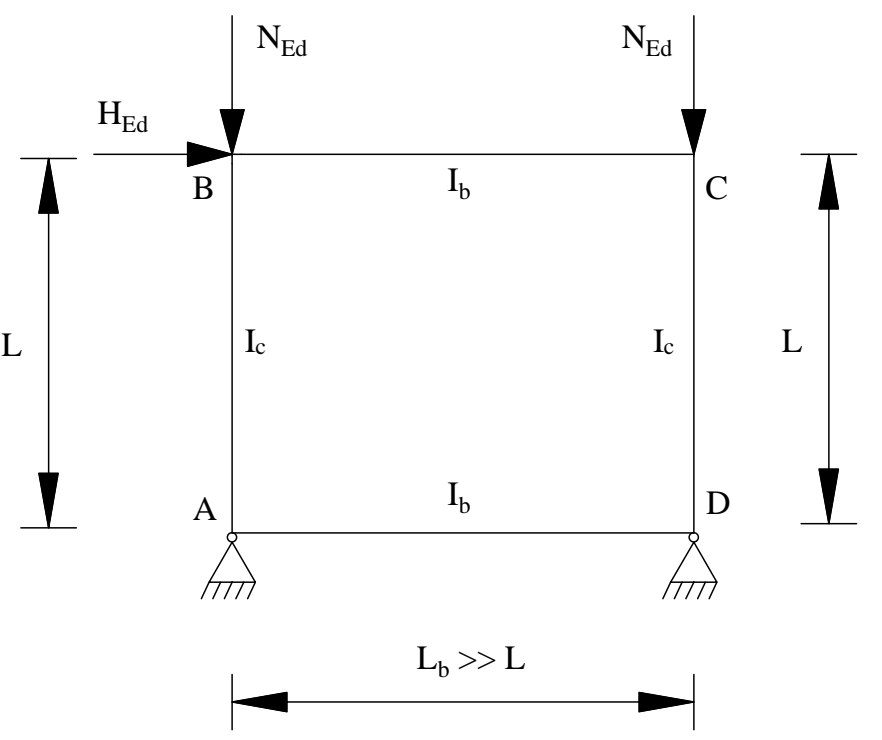

(b) FR

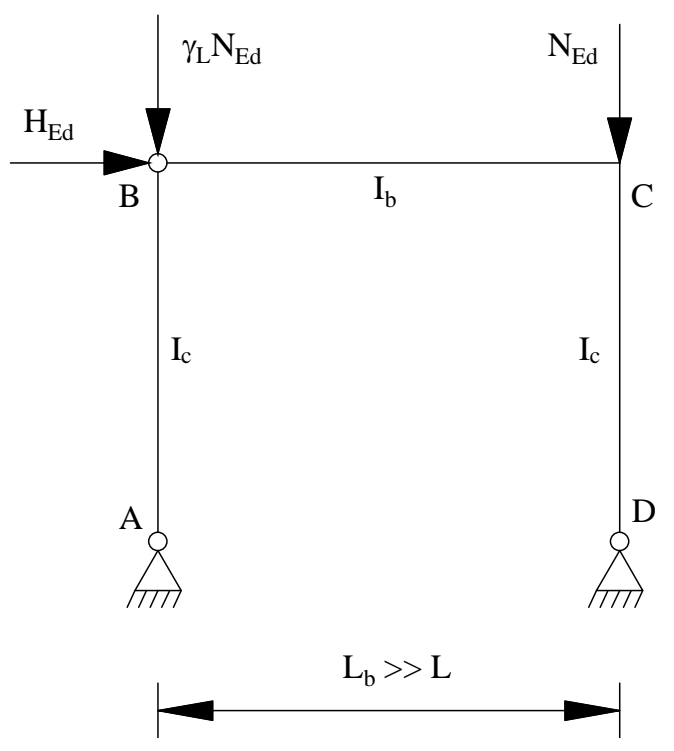

(c) LFP
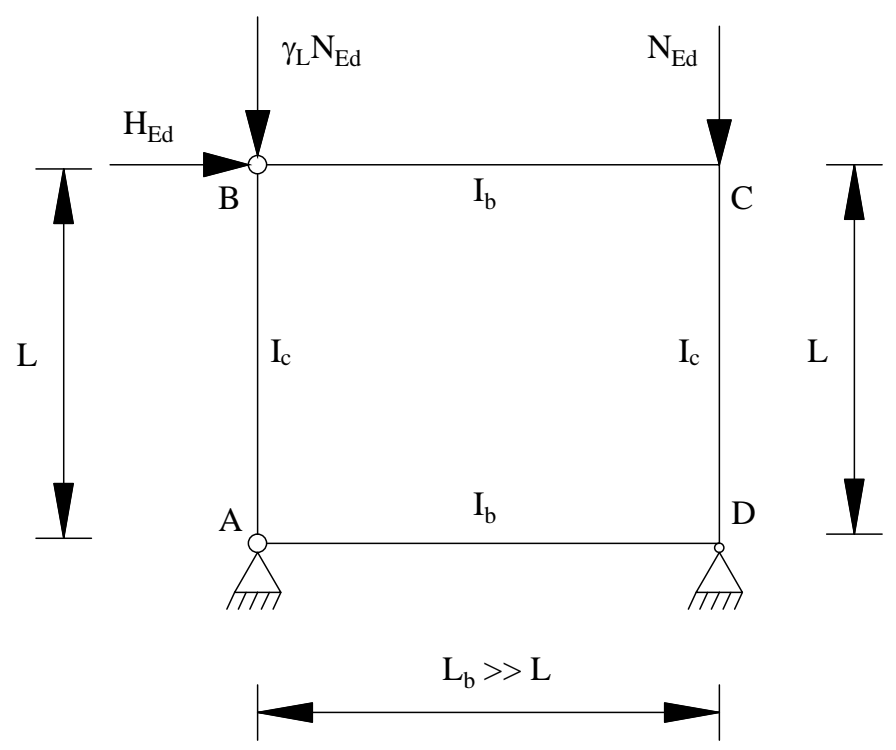

(d) LFR

Figure 4: Non-redundant benchmark frames (overturning effects are negligible $L_{b} \gg L$ ) 


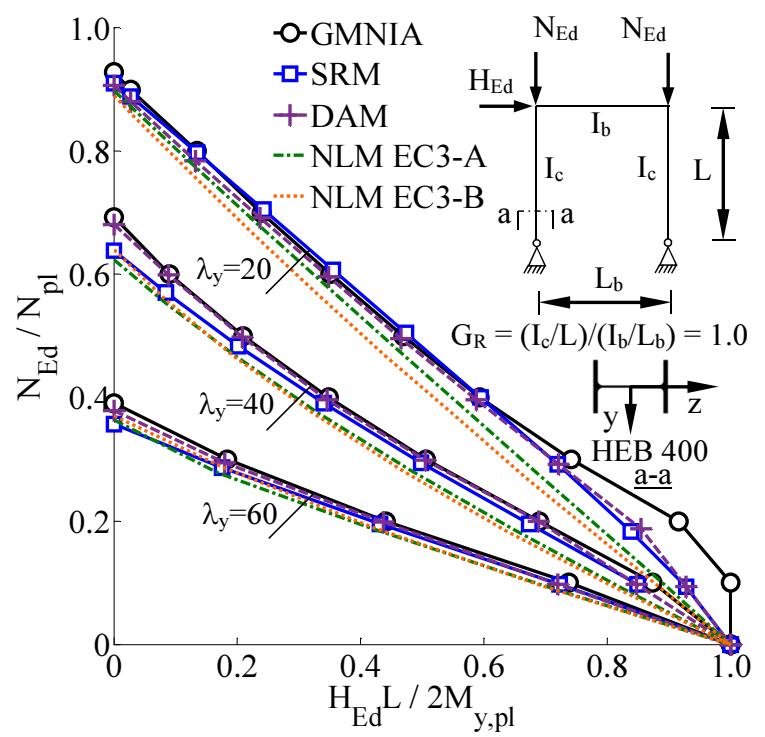

(a) $\mathrm{FP}-G_{R}=1$

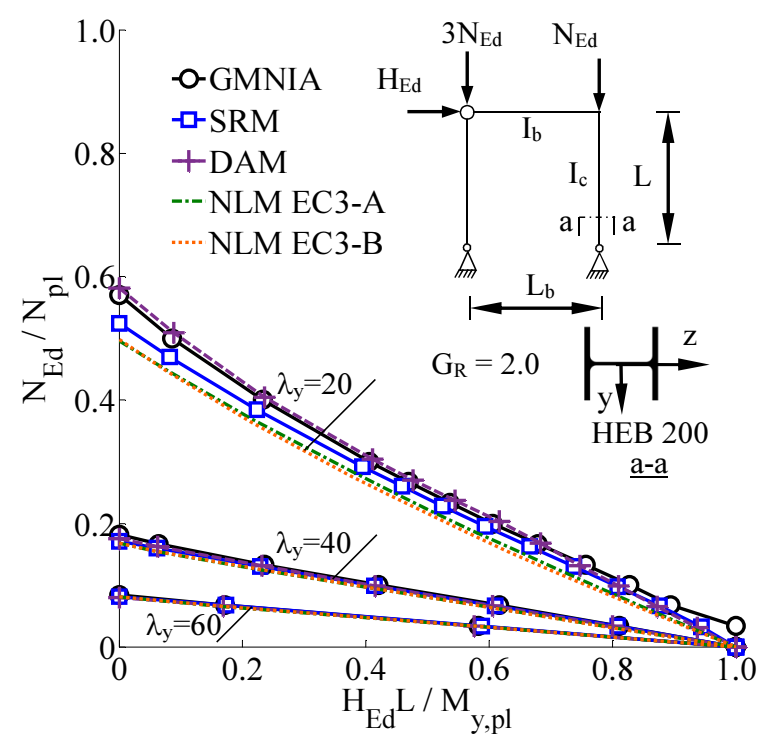

(c) LFP $-G_{R}=2, \gamma_{L}=3$

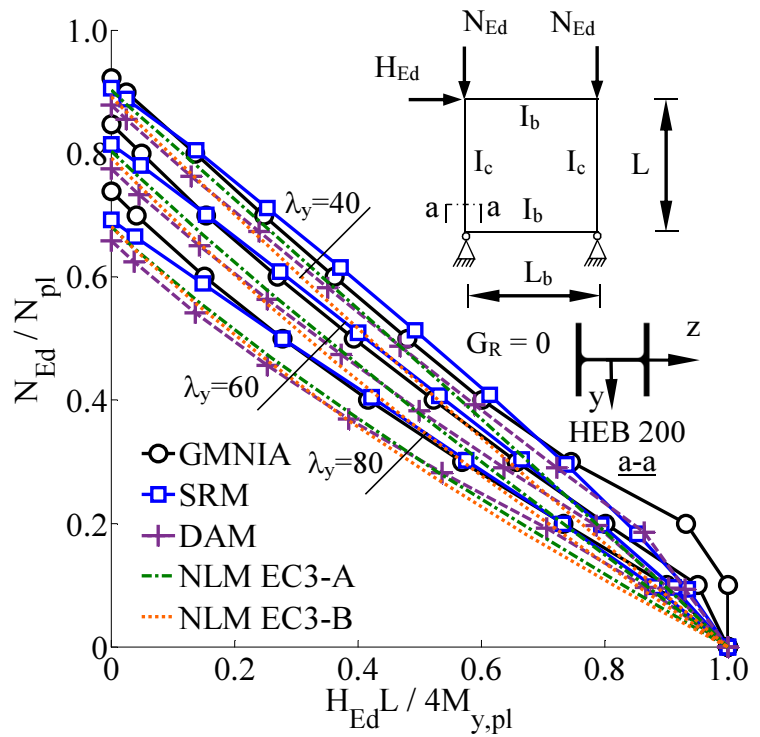

(b) $\mathrm{FR}-G_{R}=0$

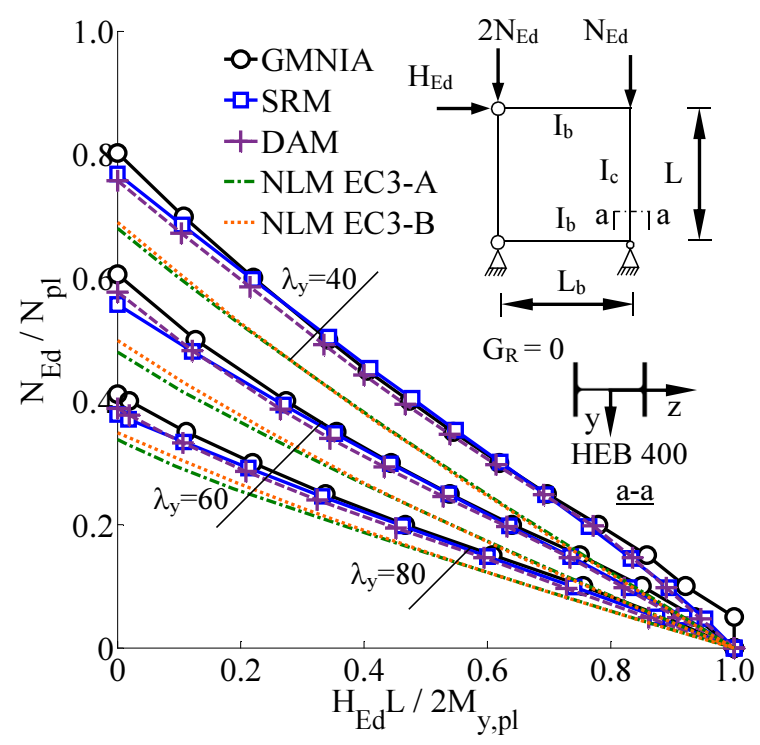

(d) LFR $-G_{R}=0, \gamma_{L}=2$

Figure 5: Comparison of the proposed stiffness reduction method (SRM) against GMNIA, the direct analysis method (DAM) and the notional load method with the Eurocode 3 Annex A (NLM EC3-A) and Annex B (NLM EC3-B) beam-column design methods for the non-redundant Kanchanalai [28] frames 


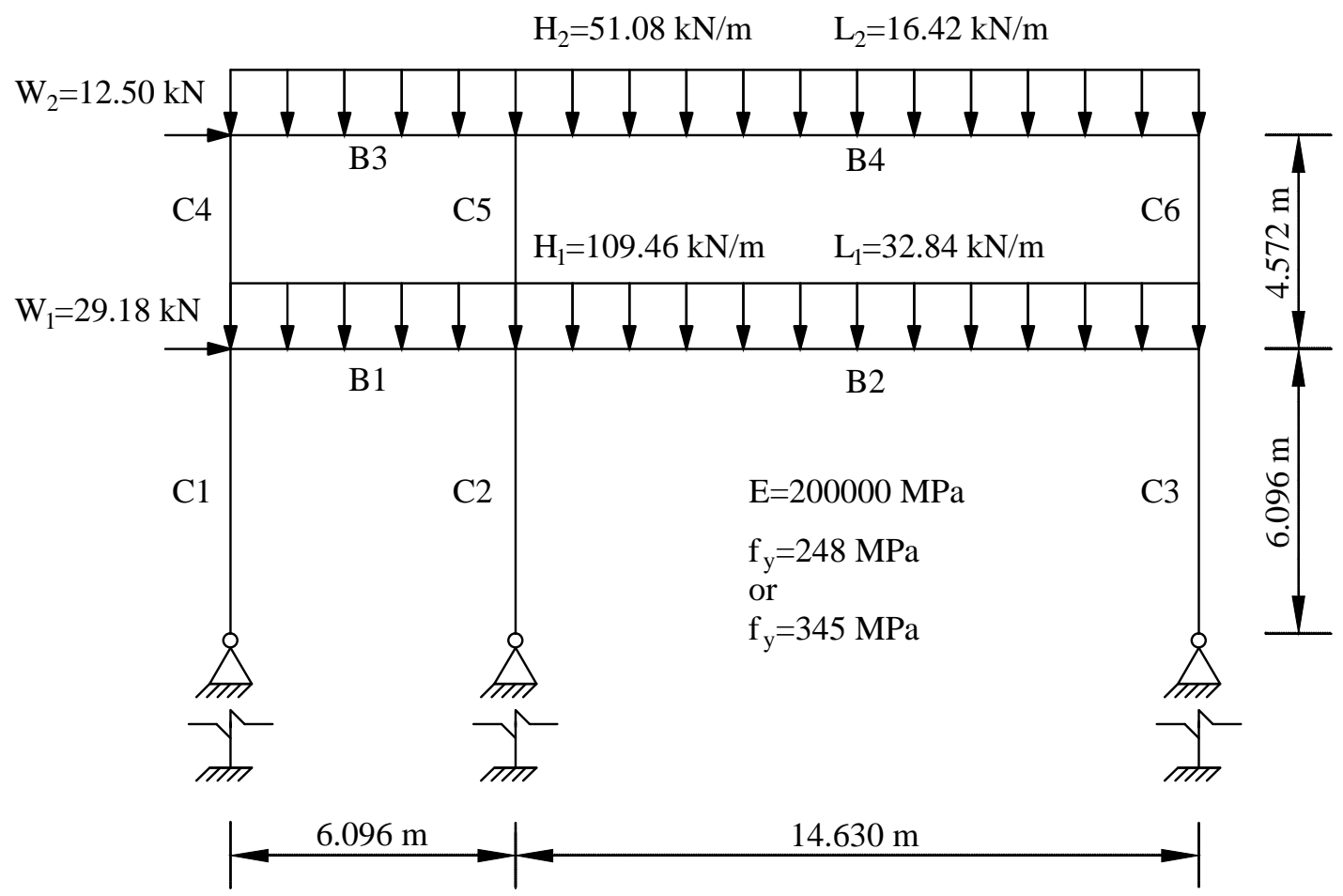

(a) Unsymmetrical frame

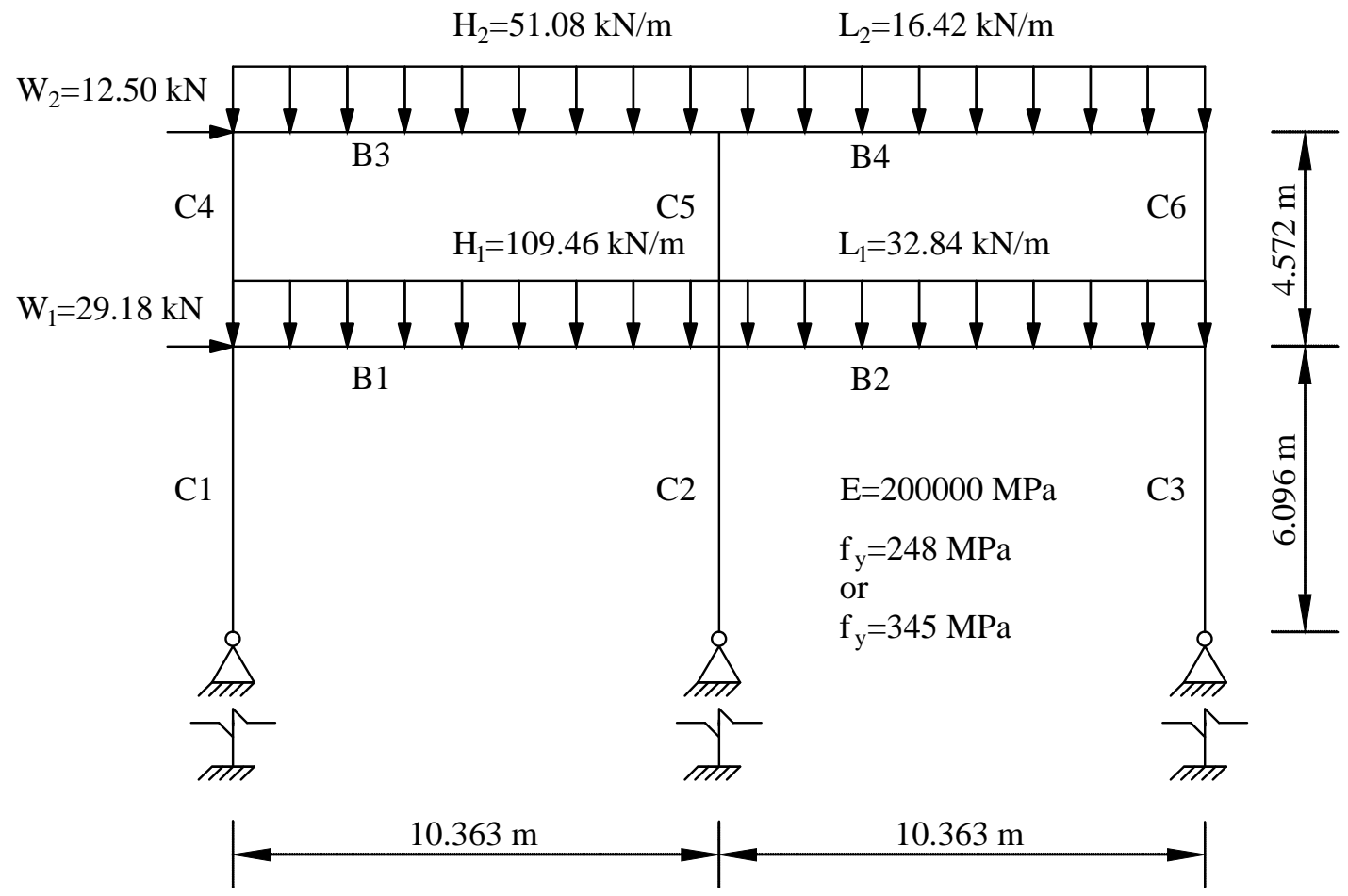

(b) Symmetrical frame

Figure 6: Geometrical properties and loading conditions of the investigated Ziemian [32] frames 


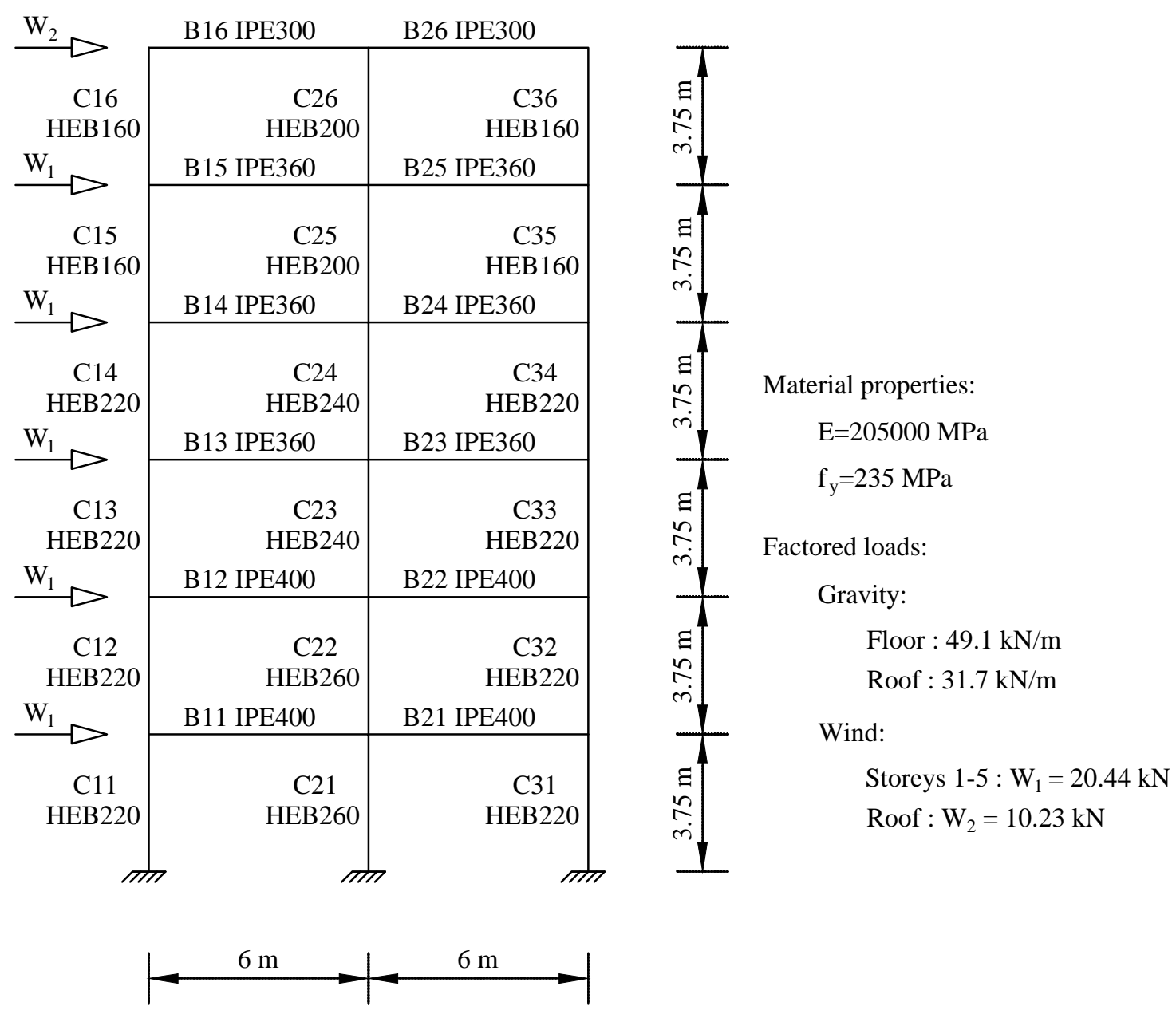

Figure 7: Geometrical and material properties and loading conditions of the modified Vogel [35] frame 